\title{
Von Neumann-Gale dynamics and capital growth in financial markets with frictions
}

\author{
Esmaeil Babaei $^{1} \cdot$ Igor V. Evstigneev $^{1}$ (D) Klaus Reiner Schenk-Hoppé ${ }^{1,2}$. \\ Mikhail Zhitlukhin ${ }^{3}$
}

Received: 9 August 2019 / Accepted: 27 December 2019 / Published online: 21 January 2020

(c) The Author(s) 2020

\begin{abstract}
The aim of this work is to extend the classical theory of growth-optimal investments (Shannon, Kelly, Breiman, Algoet, Cover and others) to models of asset markets with frictionstransaction costs and portfolio constraints. As the modelling framework, we use discrete-time dynamical systems generated by convex homogeneous multivalued operators in spaces of random vectors-von Neumann-Gale dynamical systems. The main results are concerned with the construction and characterization of investment strategies possessing properties of asymptotic growth-optimality almost surely.
\end{abstract}

Keywords Capital growth theory · Transaction costs · Benchmark strategies ·

Numeraire portfolios · Random dynamical systems · Convex multivalued operators .

Von Neumann-Gale model $\cdot$ Rapid paths

\section{Introduction}

The theory of growth-optimal investments, or capital growth theory, is a fascinating subject having a rich and peculiar history. The central question in this field is how to invest in order to

Financial support from the RFBR Grant 18-31-20031 is gratefully acknowledged.

$凶 \quad$ Igor V. Evstigneev

igor.evstigneev@manchester.ac.uk

Esmaeil Babaei

esmaeil.babaeikhezerloo@manchester.ac.uk

Klaus Reiner Schenk-Hoppé

klaus.schenk-hoppe@manchester.ac.uk

Mikhail Zhitlukhin

mikhailzh@mi-ras.ru

1 Economics, University of Manchester, Oxford Road, Manchester M13 9PL, UK

2 Department of Finance, NHH - Norwegian School of Economics, Helleveien 30, 5045 Bergen, Norway

3 Steklov Mathematical Institute of the Russian Academy of Sciences, 8 Gubkina St., Moscow 119991, Russia 
achieve the highest (asymptotic) growth rate of wealth in the long run. The first publication in this strand of literature was that by Kelly [36], who considered the case of Arrow securities (the payoff of security $i$ is 1 if the "state of the world" is $i$ and 0 otherwise) interpreted as a "horse race model". It was shown that the growth optimal investment strategy could be found by the maximization of the expected logarithm of the portfolio return: the Kelly portfolio rule. Kelly arrived at his results from information theory, and his paper was entitled "A new interpretation of information rate". The history of Kelly's discovery is described in various papers and books, including popular ones (see, e.g., Poundstone [48]). This discovery has been developed and extended by various authors, in particular by Breiman [9], Algoet and Cover [1] and Hakansson and Ziemba [32]. The paper by Algoet and Cover [1] contains the most advanced and general mathematical treatment of capital growth theory. An authoritative reference providing extensive coverage of the field is the volume [39] edited by MacLean et al. A comprehensive exposition of these questions is given in the textbook "Elements of information theory" by Cover and Thomas [14] (Chapter 16 "Information theory and portfolio theory"). An elementary introduction to the field can be found in Chapters 17 and 18 in Evstigneev et al. [25].

When speaking of those who contributed to capital growth theory, one must necessarily mention the name of Claude Shannon-the famous founder of the mathematical theory of information. Although he did not publish on investment-related issues, his ideas, expressed in his lectures on investment problems in MIT in the 1960s, strongly influenced his collaborators: Kelly, Breiman, Cover and others, who became the classics of the theory of growth optimal investments. For the history of these ideas and a related discussion see Cover [13].

Cover's [13] biographical note on Shannon mentions a discussion between Shannon and another famous scholar, mathematical economist Paul Samuelson. Cover writes:

... In the mid 1960s, Shannon gave a lecture on maximizing the growth rate of wealth and gave a geometric Wiener example.

At about this time, Shannon and Samuelson (a Nobel Prize winner-to-be in economics) held a number of evening discussion meetings on information theory and economics. It is not clear what was said in these meetings, but Samuelson seems to have become set in his views. He published several papers arguing strongly against maximizing the expected logarithm as an acceptable investment criterion. (It happens that maximizing the expected logarithm is the prescription for the growth-rate optimal portfolio.)

For example, Samuelson (1969) wrote: Our analysis enables us to dispel a fallacy that has been borrowed into portfolio theory from information theory of the Shannon type. Samuelson goes on to argue that growth rate optimal policies do not achieve maximum utility unless one has a logarithmic utility for money. Of course this is the case, but it does not deny the fact that log optimal wealth has an objective property: it has a better growth rate than that achieved by any other strategy. Since growth rate optimal policies achieve a demonstrably desirable goal, growth rate optimal portfolios should only have a utility interpretation as an afterthought. In fact, Samuelson (1979) wrote a paper entitled "Why we should not make mean log of wealth big though years to act are long." This is a two page paper in words of one syllable that makes the point that maximizing the expected log of wealth is not appropriate. The growth optimal portfolio literature has been slow to develop. It is possible that Samuelson's eloquent admonitions had their effect.

In this discussion, Samuelson and those who followed his views later presumed implicitly or explicitly that the problem of growth-optimal investments was equivalent to the problem 
of the maximization of logarithmic utility functionals. By and large this presumption was true in those models which were considered at the time of the above discussion-half a century ago. More recent studies have shown that this is not the case in more advanced and realistic models, in particular, those describing financial markets with frictions-transaction costs and trading constraints. Growth-optimal investment strategies over an infinite time horizon cannot be constructed by using a consecutive "myopic" maximization procedure step by step from $t$ to $t+1(t=0,1,2, \ldots)$.

A similar situation is characteristic for Evolutionary Finance - a rapidly developing area of research that emerged in the 2000s, see the surveys in [24,26] and an elementary introduction into the subject in [25, Chapter 20]. Evolutionary finance may be viewed as a version of the capital growth theory with endogenous equilibrium asset prices. Counterparts of the growth-optimal investment strategies in that context are the so-called unbeatable strategies [2, Sect. 6]. The problem of the identification of such strategies cannot be reduced (except for some trivial cases) to any single-agent optimization problem involving the maximization of logarithmic or any other functionals.

The objective of this paper is to extend the classical capital growth theory to models of financial markets with transaction costs and portfolio constraints. As a framework for the analysis we use von Neumann-Gale dynamical systems-a class of random dynamical systems generated by homogeneous convex set-valued operators (see the definitions in the next section). Such dynamical systems were first considered (in the deterministic case) in the context of the modeling of economic growth by von Neumann [56] and Gale [28]. Important contributions to the field were made by Radner [49], Morishima [42], Rockafellar [52], Nikaido [44], Makarov and Rubinov [40], and others. For reviews of the field see [42,44] and [40]. In our model, random states of these systems are contingent portfolios, and paths are self-financing trading strategies. The self-financing conditions are described in terms of random cones in spaces of admissible portfolio vectors. The main focus is on rapid paths, that are defined in terms of sequences of dual variables - consistent price systems, generalizing the notion of an equivalent martingale measure in the frictionless setting. Rapid paths may be regarded as counterparts of benchmark strategies (Platen [46], Platen and Heath [47]) or numeraire portfolios (Long [37]). They possess a number of important properties, in particular, it can be shown that they exhibit the fastest asymptotic growth rate of wealth with probability one.

The paper is organized as follows. Section 2 describes von Neumann-Gale dynamical systems and outlines their applications to capital growth theory. Section 3 states the main assumptions and results. Section 4 contains some lemmas needed for the proof of the main result (Theorem 1), which is conducted in Sect. 5. Section 6 establishes the property of growth-optimality of infinite rapid paths. In Sect. 7, we introduce and analyze a model of a financial market with transaction costs and portfolio constraints to which the results of this paper can be applied. The Appendix assembles some auxiliary technical results used in the paper.

\section{Von Neumann-Gale dynamics applied to finance}

Let $(\Omega, \mathcal{F}, P)$ be a complete probability space and $\mathcal{F}_{0} \subseteq \mathcal{F}_{1} \subseteq \ldots \subseteq \mathcal{F}$ a sequence of $\sigma$-algebras containing all sets in $\mathcal{F}$ of measure zero. The $\sigma$-algebra $\mathcal{F}_{t}$ is interpreted as the class of events occurring prior to time $t$. Vector functions of $\omega \in \Omega$ measurable with respect to $\mathcal{F}_{t}$ represent random vectors depending on these events. For each $t=0,1, \ldots$, 
let $X_{t}(\omega)$ be a closed pointed cone ${ }^{1}$ in an $m_{t}$-dimensional linear space $\mathbb{R}^{m_{t}}$ and for each $t=1,2, \ldots$, let $(\omega, a) \mapsto A_{t}(\omega, a)$ be a set-valued operator assigning a non-empty set $A_{t}(\omega, a) \subseteq X_{t}(\omega)$ to each $\omega \in \Omega$ and $a \in X_{t-1}(\omega)$. Throughout the paper, the following conditions of homogeneity and convexity will be imposed on the operator $A_{t}(\omega, \cdot)$. For each $\omega$, we have

$$
\lambda A_{t}(\omega, a) \subseteq A_{t}(\omega, \lambda a)
$$

for all $a \in X_{t-1}(\omega), \lambda \in[0, \infty)$ and

$$
\theta A_{t}(\omega, a)+(1-\theta) A_{t}\left(\omega, a^{\prime}\right) \subseteq A_{t}\left(\omega, \theta a+(1-\theta) a^{\prime}\right)
$$

for all $a, a^{\prime} \in X_{t-1}(\omega)$ and $\theta \in[0,1]$. (A linear combination of two sets in a vector space is the set of pairwise linear combinations of their elements.)

We will denote for shortness by $L_{\infty}\left(\mathcal{F}_{t}, \mathbb{R}^{k}\right)$ the space $L_{\infty}\left(\Omega, \mathcal{F}_{t}, P, \mathbb{R}^{k}\right)$ of essentially bounded $\mathcal{F}_{t}$-measurable functions of $\omega \in \Omega$ with values in $\mathbb{R}^{k}$. The norm $\|x(\omega)\|_{\infty}$ of a vector function $x(\omega)$ in $L_{\infty}\left(\mathcal{F}_{t}, \mathbb{R}^{k}\right)$ is defined as the essential supremum esssup $|x(\omega)|$, where $|\cdot|$ stands for the sum of the absolute values of the coordinates of a finite-dimensional vector. We say that a vector function $x(\omega)$ is a random state of the system at time $t$ and write $x \in \mathcal{X}_{t}$ if $x \in L_{\infty}\left(\mathcal{F}_{t}, \mathbb{R}^{m_{t}}\right)$ and $x(\omega) \in X_{t}(\omega)$ almost surely (a.s.). The mappings $(\omega, a) \mapsto A_{t}(\omega, a)$ generate a multivalued stochastic dynamical system. A sequence of random states $x_{0} \in \mathcal{X}_{0}, x_{1} \in \mathcal{X}_{1}, \ldots$ is called a path (trajectory) of this dynamical system if

$$
x_{t}(\omega) \in A_{t}\left(\omega, x_{t-1}(\omega)\right) \text { (a.s.). }
$$

Relation (3) can be written in the form

$$
\left(x_{t-1}(\omega), x_{t}(\omega)\right) \in Z_{t}(\omega)(\text { a.s. }),
$$

where

$$
Z_{t}(\omega)=\left\{(a, b) \in X_{t-1}(\omega) \times X_{t}(\omega): b \in A_{t}(\omega, a)\right\}
$$

is the graph of the set-valued mapping $A_{t}(\omega, \cdot)$. Clearly conditions (1) and (2) hold if and only if $Z_{t}(\omega)$ is a cone contained in $X_{t-1}(\omega) \times X_{t}(\omega)$. Since $A_{t}(\omega, a) \neq \emptyset$ for all $a \in X_{t-1}(\omega)$, the projection of $Z_{t}(\omega)$ on $X_{t-1}(\omega)$ coincides with $X_{t-1}(\omega)$. It is assumed that the cones $X_{t}(\omega)$ and $Z_{t}(\omega)$ depend $\mathcal{F}_{t}$-measurably ${ }^{2}$ on $\omega$, which means that they are determined by events occurring prior to time $t$. The dynamics of the system under consideration can equivalently be described both in terms of the mappings $A_{t}(\omega, \cdot)$ and in terms of the cones $Z_{t}(\omega)$. A sequence $x_{0} \in \mathcal{X}_{0}, x_{1} \in \mathcal{X}_{1}, \ldots$ is a path if and only if

$$
\left(x_{t-1}, x_{t}\right) \in \mathcal{Z}_{t}, t=1,2, \ldots,
$$

where

$$
\mathcal{Z}_{t}=\left\{(x, y) \in \mathcal{X}_{t-1} \times \mathcal{X}_{t}:(x(\omega), y(\omega)) \in Z_{t}(\omega)(\text { a.s. })\right\} .
$$

The classical theory of von Neumann-Gale dynamics was purely deterministic. First attempts to build its stochastic generalization were undertaken in the 1970s by Dynkin [19-21], Radner [51] and their collaborators. However, the initial attack on the problem left many questions unanswered. Substantial progress was made only in the late 1990s, and

\footnotetext{
1 A set $X$ in a linear space is called a cone if it contains with any its elements $x, y$ any non-negative linear combination $\lambda x+\mu y(\lambda, \mu \geq 0)$ of these elements. The cone $X$ is called pointed if the inclusions $x \in X$ and $-x \in X$ imply $x=0$.

${ }^{2}$ A set $A(\omega) \subseteq \mathbb{R}^{k}$ is said to depend $\mathcal{F}_{t}$-measurably on $\omega$ if the graph $\{(\omega, a): a \in A(\omega)\}$ of the set-valued mapping $\omega \mapsto A(\omega)$ belongs to the $\sigma$-algebra $\mathcal{F}_{t} \otimes \mathcal{B}\left(\mathbb{R}^{k}\right)$, where $\mathcal{B}(\cdot)$ stands for the Borel $\sigma$-algebra.
} 
final solutions to the main open problems were obtained only in the 2000s — see [23] and references therein.

At about the same time it was observed [18] that stochastic analogues of von NeumannGale dynamical systems provide a natural and convenient framework for the modeling of financial markets with frictions - transaction costs and portfolio constraints. This observation not only gave a new momentum to studies in the field and posed new interesting questions, but also made it possible to find a key to the solution of old problems. The new, financial interpretation of the mathematical notions and objects at hand amazingly suggested the way of proofs in [23] that could not be found earlier.

A central goal in the theory of von Neumann-Gale dynamics is to single out and investigate a class of trajectories that grow faster in a certain sense than others. The key notion here is that of a rapid path. To give its definition let us first define the important notion of a dual path. Let $X_{t}^{*}(\omega)$ denote the dual cone of $X_{t}(\omega)$ :

$$
X_{t}^{*}(\omega)=\left\{p \in \mathbb{R}^{m_{t}}: p a \geq 0, a \in X_{t}(\omega)\right\},
$$

where $p a$ is the scalar product of the vectors $p$ and $a$ in $\mathbb{R}^{m_{t}}$. For shortness, we will use the notation $L^{1}\left(\mathcal{F}_{t}, \mathbb{R}^{k}\right)$ for the space $L^{1}\left(\Omega, \mathcal{F}_{t}, P, \mathbb{R}^{k}\right)$ of integrable $\mathcal{F}_{t}$-measurable vector functions with values in $\mathbb{R}^{k}$. A dual path (dual trajectory) is a sequence of vector functions $p_{1}(\omega), p_{2}(\omega), \ldots$ such that for all $t=1,2, \ldots$ we have $p_{t} \in L^{1}\left(\mathcal{F}_{t}, \mathbb{R}^{m_{t-1}}\right)$,

$$
p_{t}(\omega) \in X_{t-1}^{*}(\omega) \text { (a.s.), }
$$

and for almost all $\omega$,

$$
\bar{p}_{t+1}(\omega) b \leq p_{t}(\omega) a \text { for all }(a, b) \in Z_{t}(\omega) .
$$

here, $\bar{p}_{t+1}(\omega):=E_{t} p_{t+1}(\omega)$ and $E_{t}(\cdot)=E\left(\cdot \mid \mathcal{F}_{t}\right)$ is the conditional expectation given $\mathcal{F}_{t}$. Denote by $Z_{t}^{\times}(\omega)$ the cross-dual cone for $Z_{t}(\omega)$ :

$$
Z_{t}^{\times}(\omega)=\left\{(c, d) \in \mathbb{R}^{m_{t-1}} \times \mathbb{R}^{m_{t}}: d b-c a \leq 0 \text { for all }(a, b) \in Z_{t}(\omega)\right\} .
$$

The definition of a dual path can be reformulated as follows. This is a sequence $p_{1}(\omega), p_{2}(\omega), \ldots$ such that for all $t=1,2, \ldots$, we have $p_{t} \in L^{1}\left(\mathcal{F}_{t}, \mathbb{R}^{m_{t-1}}\right)$, condition (8) holds and

$$
\left(p_{t}(\omega), \bar{p}_{t+1}(\omega)\right) \in Z_{t}^{\times}(\omega) \text { (a.s.). }
$$

Let us say that a dual path $p_{1}, p_{2}, \ldots$ supports a path $x_{0}, x_{1}, \ldots$ if

$$
p_{t+1} x_{t}=1 \text { (a.s.) }
$$

for all $t=0,1, \ldots$. A trajectory is called rapid if there exists a dual trajectory supporting it. What matters in (12) is that $p_{t+1} x_{t}$ is a strictly positive constant (independent of time and random factors). The value 1 for this constant is chosen only for the sake of convenience.

The term "rapid" is motivated, in particular, by the fact that for each $t=1,2, \ldots$.

$$
E_{t} \frac{p_{t+1} y_{t}}{p_{t} y_{t-1}}=\frac{\bar{p}_{t+1} y_{t}}{p_{t} y_{t-1}} \leq \frac{\bar{p}_{t+1} x_{t}}{p_{t} x_{t-1}}=1 \text { (a.s.) }
$$

for all paths $y_{0}, y_{1}, \ldots$ with $p_{t} y_{t-1}>0$ (a.s.). This means that the path $x_{0}, x_{1}, \ldots$ maximizes the conditional expectation given $\mathcal{F}_{t}$ of the growth rate $p_{t+1} y_{t} / p_{t} y_{t-1}$ over each time period $(t-1, t]$, the maximum being equal to 1 . The growth rate is measured in terms of the dual variables $p_{t}$, which in economic and financial applications typically represent prices.

Rapid paths may be viewed as a generalization of benchmark strategies (Platen [46], Platen and Heath [47]) or numeraire portfolios (Long [37]). Their idea goes back to the notion of 
a competitive path in models of economic dynamics: such paths maximize profits over each time period $(t-1, t)$ —see Malinvaud [41], Radner [50], Gale [29], Peleg [45], Dasgupta and Mitra [16], and especially the paper by Clark [11] linking this strand of literature to finance and containing further references.

Dual paths are analogous to consistent price systems, generalizing the concept of an equivalent martingale measure involved in classical no-arbitrage criteria (Jouini and Kallal [38], Cvitanić and Karatzas [15], Schachermayer [54], Guasoni et al. [31], Kabanov and Safarian [34] and others).

Let us outline a model of this kind. At each time $t=0,1,2, \ldots$, there are $m_{t}$ assets in the market. A (contingent) portfolio of assets is an $\mathcal{F}_{t}$-measurable random vector $y_{t}(\omega)=$ $\left(y_{t}^{1}(\omega), \ldots, y_{t}^{m_{t}}(\omega)\right)$. Those portfolios $y_{t}$ for which $y_{t}(\omega) \in X_{t}(\omega)$ (a.s.) are admissible at time $t$. An investment/trading strategy is a sequence of admissible portfolios $\left(y_{t}\right)_{t \geq 0}$. The main focus is on self-financing strategies defined by the condition

$$
\left(y_{t-1}(\omega), y_{t}(\omega)\right) \in Z_{t}(\omega) \text { (a.s.), }
$$

where $Z_{t}(\omega) \subseteq \mathbb{R}^{m_{t-1}} \times \mathbb{R}^{m_{t}}$ is the given closed cone depending $\mathcal{F}_{t}$-measurably on $\omega$. The cones $Z_{t}(\omega), t=1,2, \ldots$, define the self-financing constraints. Condition (14) states in general terms that the portfolio $y_{t-1}$ can be transformed to $y_{t}$ by buying and selling assets under transaction costs (see an example in (15) below). Self-financing strategies are nothing but paths in the von Neumann-Gale dynamical system with the transition cones $Z_{t}(\omega)$. We will deal only with such strategies, and so in what follows, "self-financing" will be omitted.

A basic example of the transition cone $Z_{t}(\omega)$ in the financial market model with (proportional) transaction costs can be described as follows. Let $\underline{S}_{t}^{i}(\omega)<\bar{S}_{t}^{i}(\omega)$ be the vectors of the asset $i$ 's bid and ask prices: you pay $\bar{S}_{t}^{i}(\omega)$ when you buy and you get $\underline{S}_{t}^{i}(\omega)$ when you sell. The cone $Z_{t}(\omega)$ consists of pairs of portfolios $(x, y)$ satisfying

$$
\sum_{i=1}^{m} \bar{S}_{t}^{i}\left(y^{i}-x^{i}\right)_{+} \leq \sum_{i=1}^{m} \underline{S}_{t}^{i}\left(x^{i}-y^{i}\right)_{+},
$$

where $a_{+}:=\max \{a, 0\}$. It is assumed here that the number of assets $m_{t}$ does not depend on $t: m_{t}=m$. The inequality (15) means that asset purchases are made only at the expense of sales of available assets (under transaction costs).

Questions of asset pricing and hedging in the above basic framework were considered in the seminal paper by Jouini and Kallal [38]. Kabanov [35] proposed a geometric approach that made it possible to extend the theory to models allowing direct (unmediated by cash) trades between assets, and therefore, applicable to currency markets. We refer the reader to the book by Kabanov and Safarian [34] for a detailed review of this area of research. For more recent work see the paper [27] and references therein.

Important examples of the portfolio admissibility constraints are given by margin requirements, that are present in one form or another in all real-world financial markets. Only those portfolios $x=\left(x^{1}, \ldots, x^{m}\right)$ are regarded as admissible for which

$$
M \sum_{i=1}^{m} \bar{S}_{t}^{i}\left(-x^{i}\right)_{+} \leq \sum_{i=1}^{m} \underline{S}_{t}^{i}\left(x^{i}\right)_{+},
$$

where $M>1$ is some constant (margin). Condition (16) means that an admissible portfolio can always be liquidated in such a way that its long positions would compensate its short positions with excess, as specified in (16). The purpose of such requirements is to exclude (or at least to reduce) the possibility of the investor's bankruptcy as a result of sudden price 
jumps. Clearly property (16) automatically guarantees the absence of arbitrage opportunities, which makes superfluous any considerations involving the no-arbitrage hypothesis as such.

In the above example it is assumed that portfolio positions are measured in terms of ("physical") units of assets. However, it is often more convenient to specify positions of a portfolio in monetary terms. In models of this kind, the coordinates $x^{i}$ and $y^{i}$ of portfolio vectors $x$ and $y$ indicate the values of asset holdings at time $t$ expressed in terms of the current market prices $S_{t}^{i}$. The transition cone $Z_{t}(\omega)$ consists of pairs of portfolios $(x, y)$ such that

$$
\sum_{i=1}^{m}\left(1+\lambda_{t, i}^{-}\right)\left(y^{i}-\frac{S_{t}^{i}}{S_{t-1}^{i}} x^{i}\right)_{+} \leq \sum_{i=1}^{m}\left(1-\lambda_{t, i}^{+}\right)\left(\frac{S_{t}^{i}}{S_{t-1}^{i}} x^{i}-y^{i}\right)_{+},
$$

where $\lambda_{t, i}^{-}(\omega) \geq 0$ and $1>\lambda_{t, i}^{+}(\omega) \geq 0$ are the transaction cost rates for buying and selling asset $i$, respectively. The inequality in (17), as well as in (15), expresses the self-financing condition, meaning that purchases of assets can be made only at the expense of sales of other assets. The simplest margin requirements in this setting take on the following form:

$$
M \sum_{i=1}^{m} x_{-}^{i} \leq \sum_{i=1}^{m} x_{+}^{i}
$$

If portfolio positions are specified in monetary terms, then the variables $p_{t}^{i}$ constituting a dual path may be interpreted as market consistent discount factors.

The framework in which the transition cones $Z_{t}(\omega)$ are described in terms of portfolio values has the following advantage. Practically all models that can be used for practical computations are based on some assumptions of stationarity: "tomorrow" must to some extent resemble "today". In the framework of (17), a natural assumption of this kind is that the process of asset returns $\left(S_{t}^{i}-S_{t-1}^{i}\right) / S_{t-1}^{i}$ is stationary, which is a common hypothesis in finance (lying in the basis, e.g., of the Black-Scholes formula). Analogous conditions for (15) would mean stationarity of the price process - a less plausible hypothesis, having a number of implications that might be viewed as paradoxical (e.g. the phenomenon of "volatility-induced growth", see [17] and [25, Sect. 18.4]).

A detailed description and analysis of a financial market model of the above type is given in Sect. 7.

\section{Assumptions and results}

Let us formulate the assumptions that will be used in this paper. Let $|\cdot|$ denote the norm of a vector in a finite-dimensional space defined as the sum of the absolute values of its coordinates. For a finite-dimensional vector $a$, we will denote by $\mathbb{B}(a, r)$ the ball $\{b:|b-a| \leq r\}$.

Let us introduce the following conditions.

(A1) For every $t=0,1, \ldots$, there exists an $\mathcal{F}_{t}$-measurable random vector $q_{t}(\omega) \in X_{t}^{*}(\omega)$ satisfying

$$
H_{t}(\omega)^{-1}|a| \leq q_{t}(\omega) a \leq H_{t}(\omega)|a|, a \in X_{t}(\omega), \omega \in \Omega,
$$

where $H_{t}(\omega) \geq 1$ is an $\mathcal{F}_{t}$-measurable function with $E \ln H_{t}(\omega)<\infty$. This condition implies, in particular, that the cone $X_{t}(\omega)$ is pointed.

(A2) For every $t=1,2, \ldots, \omega \in \Omega$ and $a \in X_{t-1}(\omega)$, there exists $b \in X_{t}(\omega)$ such that $(a, b) \in Z_{t}(\omega)$. 
(A3) There exist constants $K_{t}(t=1,2, \ldots)$ such that $|b| \leq K_{t}|a|$ for any $(a, b) \in Z_{t}(\omega)$ and $\omega \in \Omega$.

(A4) For each $t=1,2, \ldots$, there exists a bounded $\mathcal{F}_{t}$-measurable vector function $\stackrel{\circ}{z}_{t}=$ $\left(\stackrel{\circ}{x}_{t}, \stackrel{\circ}{y}_{t}\right)$ such that for all $\omega \in \Omega$, we have

$$
\left(\stackrel{\circ}{x}_{t}(\omega), \stackrel{\circ}{y}_{t}(\omega)\right) \in Z_{t}(\omega) \text {, }
$$

and

$$
\mathbb{B}\left(\stackrel{\circ}{y}_{t}(\omega), \varepsilon_{t}\right) \subseteq X_{t}(\omega),
$$

where $\varepsilon_{t}>0$ is some constant.

Theorems 1 and 2 we formulate below hold under the assumptions (A1)-(A4).

Theorem 1 Let assumptions (A1)-(A4) hold. Let $x_{0}(\omega)$ be a vector function in $\mathcal{X}_{0}$ such that $\mathbb{B}\left(x_{0}(\omega), \delta\right) \subseteq X_{0}(\omega)$ for some constant $\delta>0$. There exists an infinite rapid path with initial state $x_{0}$.

This result generalizes to general cones $X_{t}(\omega)$ the analogous result in [8] pertaining to the case, where $X_{t}(\omega)$ are the standard non-negative cone $\mathbb{R}_{+}^{m_{t}}$.

The proof of Theorem 1 is based on a previous result [7, Theorem 1] on finite rapid paths. We formulate it as Theorem 2 below. The versions of the basic definitions for a finite time horizon are as follows. Let $N>1$ be a natural number. A path over the finite time horizon from 0 to $N$ is a sequence $x_{0} \in \mathcal{X}_{0}, \ldots, x_{N} \in \mathcal{X}_{N}$ satisfying (6) for all $t=1, \ldots, N$. A dual path $p_{1}, p_{2}, \ldots, p_{N+1}$ is a sequence of vector functions such that

$$
\begin{aligned}
& p_{1} \in L^{1}\left(\mathcal{F}_{1}, \mathbb{R}^{m_{0}}\right), \ldots, p_{N} \in L^{1}\left(\mathcal{F}_{N}, \mathbb{R}^{m_{N-1}}\right), p_{N+1} \in L^{1}\left(\mathcal{F}_{N}, \mathbb{R}^{m_{N}}\right), \\
& p_{t}(\omega) \in X_{t-1}^{*}(\omega) \text { (a.s.), } t=1,2, \ldots, N+1,
\end{aligned}
$$

and

$$
\left(p_{t}(\omega), \bar{p}_{t+1}(\omega)\right) \in Z_{t}^{\times}(\omega)(\text { a.s. }), t=1, \ldots, N .
$$

A dual path $p_{1}, p_{2}, \ldots, p_{N+1}$ supports a path $x_{0}, x_{1}, \ldots, x_{N}$ if (12) holds for $t=0, \ldots, N$. A trajectory $x_{0}, x_{1}, \ldots, x_{N}$ is called rapid if there exists a dual trajectory $p_{1}, p_{2}, \ldots, p_{N+1}$ supporting it.

Theorem 2 Let $x_{0}(\omega)$ be a vector function in $\mathcal{X}_{0}$ such that $\mathbb{B}\left(x_{0}(\omega), \delta\right) \subseteq X_{0}(\omega)$ for some constant $\delta>0$. For each $N \geq 1$, there exists a finite rapid path $x_{0}, \ldots, x_{N}$ with the initial state $x_{0}$.

This theorem extends to general random cones $X_{t}(\omega)$ earlier results obtained in [22] (also for the finite-horizon case) in a setting where $X_{t}(\omega)=\mathbb{R}_{+}^{m_{t}}$.

Remark 1 To prove Theorem 1 we construct an infinite rapid path by passing to the limit from finite ones, whose existence is stated in Theorem 2. The latter theorem is proved in [7, Theorem 1] under the assumptions (A1)-(A4). However, not all of these assumptions are needed to deduce the former result from the latter. In the course of the proof of Theorem 1 given in Sect. 3, we rely only upon conditions (A3) and (A4) and the fact that the cones $X_{t}(\omega)$ are pointed; assumptions (A1) and (A2) are not used.

An important property of infinite rapid paths is their asymptotic growth-optimality holding under a fairly general assumption (A5) we formulate below. Let us say that a path $\left(x_{t}\right)_{t=0}^{\infty}$ is asymptotically growth-optimal if for any other path $\left(x_{t}^{\prime}\right)_{t=0}^{\infty}$ there exists a supermartingale $\left(\xi_{t}\right)_{t=1}^{\infty}$ such that $\left|x_{t}^{\prime}\right| /\left|x_{t}\right| \leq \xi_{t}$. 
We introduce following condition (A5).

(A5) There exist a real number $\gamma>0$ and a natural number $l$ such that for every $t \geq 0$ and every random vector $y_{t} \in \mathcal{X}_{t}$, one can find random vectors $y_{t+1} \in \mathcal{X}_{t+1}, \ldots, y_{t+l} \in \mathcal{X}_{t+l}$ satisfying with probability one

$$
\left(y_{t}(\omega), y_{t+1}(\omega)\right) \in Z_{t+1}(\omega), \ldots,\left(y_{t+l-1}(\omega), y_{t+l}(\omega)+y(\omega)\right) \in Z_{t+l}(\omega)
$$

for all $y \in L_{\infty}\left(\mathcal{F}_{t+l}, \mathbb{R}^{m_{t+l}}\right)$ with $|y(\omega)| \leq \gamma\left|y_{t}(\omega)\right|$.

Theorem 3 Let condition (A5) and condition (A3) with a constant $K_{t}=K$ independent of $t$ hold. Let $m_{t} \leq m$ where $m$ is some fixed number. Then any rapid path is asymptotically growth-optimal.

The property of asymptotic optimality, as defined above, has the following important implications. If $\left|y_{t}\right| /\left|x_{t}\right| \leq \xi_{t}, t=1,2 \ldots$ (a.s.), where $\xi_{t}$ is a supermartingale, the following assertions hold.

(a) With probability one

$$
\sup _{t} \frac{\left|y_{t}\right|}{\left|x_{t}\right|}<\infty
$$

i.e. no strategy can grow asymptotically faster than $x_{0}, x_{1}, \ldots$ (a.s.).

(b) The strategy $x_{0}, x_{1}, \ldots$ a.s. maximizes the exponential growth rate

$$
\lim \sup _{t \rightarrow \infty} \frac{1}{t} \ln \left|x_{t}\right|
$$

(c) We have

$$
\sup _{t} E \frac{\left|y_{t}\right|}{\left|x_{t}\right|}<\infty \text { and } \sup _{t} E \ln \frac{\left|y_{t}\right|}{\left|x_{t}\right|}<\infty,
$$

and moreover, $\sup _{t}$ in (24) can be replaced by $\sup _{\tau}$ where $\tau$ ranges through the set of all stopping times with respect to the filtration $\mathcal{F}_{0} \subseteq \mathcal{F}_{1} \subseteq \ldots \subseteq \mathcal{F}$.

Assertion (a) follows from a.s. convergence of non-negative supermartingales; (b) is immediate from (a); the first part of (c) holds because $\xi_{t}$ is a non-negative supermartingale; the second part of (c) is obtained by using Jensen's inequality and the supermartingale property: $E\left(\ln \xi_{t+1} \mid \mathcal{F}_{t}\right) \leq \ln E\left(\xi_{t+1} \mid \mathcal{F}_{t}\right) \leq \ln \xi_{t}$. The possibility of replacing $\sup _{t}$ by $\sup _{\tau}$ in (24) follows from Doob's optional sampling theorem (see, e.g., Grimmett and Stirzaker [30], pp. 491-495).

Note that the above properties (a)-(c) remain valid if $\left|x_{t}\right|$ and $\left|y_{t}\right|$ are replaced by $\phi_{t}\left(\omega, x_{t}\right)$ and $\phi_{t}\left(\omega, y_{t}\right)$ respectively with any function $\phi_{t}(\omega, b)$, possibly random and depending on $t$, which satisfies the following condition $(\mathbf{L})$.

(L) There exist non-random constants $0<s \leq S$ such that $s|b| \leq \phi_{t}(\omega, b) \leq S|b|$ for all $t, \omega$ and $b \in X_{t}(\omega)$.

\section{Three lemmas}

We begin with three simple lemmas needed for the proof of Theorem 1 .

Lemma 1 Let $X$ be a cone in $\mathbb{R}^{k}, d$ an element in $X^{*}$, and $y$ a vector in $X$ such that $\mathbb{B}(y, \varepsilon) \subseteq X$. Then

$$
d y \geq \frac{\varepsilon}{k}|d| .
$$


Proof We have $d(y-h) \geq 0$ for any $h$ with $|h| \leq \varepsilon$. Consequently,

$$
d y \geq \max _{|h| \leq \varepsilon} d h=\varepsilon \max _{i}\left|d^{i}\right| \geq \frac{\varepsilon}{k}|d| \quad\left[d=\left(d^{1}, \ldots, d^{k}\right)\right],
$$

which yields (25).

Put

$$
C_{t}:=\frac{m_{t}\left\|\stackrel{\circ}{x}_{t}\right\|_{\infty}}{\varepsilon_{t}}(t \geq 1), C_{0}:=m_{0} \delta^{-1},
$$

where $\stackrel{\circ}{t}_{t}$ is defined in (A4) and

$$
C^{t}:=C_{t-1} \ldots C_{1} C_{0}(t \geq 1)
$$

where $\delta$ is the constant for which $B\left(x_{0}(\omega), \delta\right) \subseteq X_{0}(\omega)$. Define

$$
K^{t}:=K_{t} \ldots K_{1}\left\|x_{0}\right\|_{\infty}(t \geq 1) .
$$

Lemma 2 Let $\left(x_{t}\right)_{t=0}^{N}$ be a rapid path and $\left(p_{t}\right)_{t=1}^{N+1}$ a dual path supporting it $(1 \leq N \leq \infty)$. Then

$$
\left|x_{t}\right| \leq K^{t} \text { (a.s.), }
$$

for $t=0,1, \ldots, N$ and

$$
E\left|p_{t}\right| \leq C^{t}
$$

for $t=1, \ldots, N+1$.

Proof Inequality (27) is immediate from (A3). To prove (28) we write

$$
|| \stackrel{\circ}{x}_{t} \|_{\infty} E\left|p_{t}\right| \geq E\left|p_{t}\right|\left|\stackrel{\circ}{x}_{t}\right| \geq E p_{t} \stackrel{\circ}{x}_{t} \geq E \bar{p}_{t+1} \stackrel{\circ}{y}_{t}=E p_{t+1} \stackrel{\circ}{y}_{t} \geq E \frac{\varepsilon_{t}}{m_{t}}\left|p_{t+1}\right|
$$

$(t \geq 1)$. Here the third inequality holds because $\left(p_{t}(\omega), \bar{p}_{t+1}(\omega)\right) \in Z_{t}^{\times}(\omega)$ (a.s.) and $\left(\stackrel{\circ}{x}_{t}(\omega), \stackrel{\circ}{y}_{t}(\omega)\right) \in Z_{t}(\omega)$. The last inequality is valid by virtue Lemma 1 since $p_{t+1}(\omega) \in$ $X_{t}^{*}(\omega)$ (a.s.) and $\mathbb{B}\left(\dot{y}_{t}(\omega), \varepsilon_{t}\right) \subseteq X_{t}(\omega)$. From (29) and (26) we get $E\left|p_{t+1}\right| \leq C_{t} E\left|p_{t}\right|$ $(t \geq 1)$ and it remains to observe that $E\left|p_{1}\right| \leq m_{0} \delta^{-1}$. Indeed, by virtue of (25),

$$
1=p_{1} x_{0} \geq \frac{\delta}{m_{0}}\left|p_{1}\right| \text { (a.s.) }
$$

because $p_{1}(\omega) \in X_{0}^{*}(\omega)$ (a.s.) and $\mathbb{B}\left(x_{0}(\omega), \delta\right) \subseteq X_{0}(\omega)$. Consequently,

$$
\left|p_{1}\right| \leq m_{0} \delta^{-1}
$$

which completes the proof.

Lemma 3 For each $t=1,2, \ldots$ and for almost all $\omega$, if

$$
(c, d) \in Z_{t}^{\times}(\omega) \text { and } d \in X_{t}^{*}(\omega),
$$

then

$$
|d| \leq C_{t}|c|
$$


Proof This follows from the inequalities

$$
|c||| \stackrel{\circ}{x}_{t} \|_{\infty} \geq|c|\left|\stackrel{\circ}{x}_{t}(\omega)\right| \geq c \stackrel{\circ}{x}_{t}(\omega) \geq d \stackrel{\circ}{y}_{t}(\omega) \geq \frac{\varepsilon_{t}}{m_{t}}|d|
$$

holding for almost all $\omega$ and all $(c, d)$ satisfying (32). Note that the third inequality in (34) is valid because $(c, d) \in Z_{t}^{\times}(\omega)$ and $\left(\stackrel{\circ}{x}_{t}(\omega), \stackrel{\circ}{y}_{t}(\omega)\right) \in Z_{t}(\omega)$, and the fourth is a consequence of Lemma 1.

Remark 2 We point to a distinction between Lemmas 2 and 3. Relations (28) and (29) hold in terms of expectations while (33) and (34) are valid for almost all $\omega$ and all $(c, d)$ satisfying (32). It might seem that the former lemma is a direct consequence of the latter, but one has to be cautious here: we know that $\left(p_{t}(\omega), \bar{p}_{t+1}(\omega)\right) \in Z_{t}^{\times}(\omega)$ (a.s.), but it is not assumed that $\left(p_{t}(\omega), p_{t+1}(\omega)\right) \in Z_{t}^{\times}(\omega)$ (a.s.).

\section{Proof of the main result}

Denote by $\mathcal{P}_{t}$ the set of $\mathcal{F}_{t}$-measurable $m_{t-1}$-dimensional vector functions $p_{t}(\omega)$ such that $E\left|p_{t}(\omega)\right|<\infty$ and $p_{t}(\omega) \in X_{t-1}^{*}(\omega)$ (a.s.). Let $\mathcal{Q}_{t}$ be the set of $\mathcal{F}_{t}$-measurable $m_{t^{-}}$ dimensional vector functions $q_{t}(\omega)$ such that $E\left|q_{t}(\omega)\right|<\infty$ and $q_{t}(\omega) \in X_{t}^{*}(\omega)$ (a.s.).

To alleviate notation we will often omit " $\omega$ " when this does not lead to ambiguity.

Proof of Theorem 1 By virtue of Theorem 2, for each natural number $N$ there exist a finite rapid path $x_{0}(N), \ldots, x_{N}(N)$ with $x_{0}(N)=x_{0}$ and a dual path $p_{1}(N), \ldots, p_{N+1}(N)$ supporting it.

We will construct by induction for each $t=1,2, \ldots$ a triplet of vector functions

$$
\left(x_{t}, p_{t}, q_{t}\right) \in \mathcal{X}_{t} \times \mathcal{P}_{t} \times \mathcal{Q}_{t}
$$

such that

$$
\begin{aligned}
& \left(p_{t}, q_{t}\right) \in Z_{t}^{\times}(\text {a.s. }), \\
& \left(x_{t-1}, x_{t}\right) \in Z_{t}(\text { a.s. }), \\
& p_{t} x_{t-1}=1 \text { (a.s.), } \\
& q_{t-1}-E_{t-1} p_{t} \in X_{t-1}^{*}(\omega) \text { (a.s.), if } t \geq 2,
\end{aligned}
$$

and a sequence of integer-valued $\mathcal{F}_{t}$-measurable random variables

$$
t<N_{1}^{t}<N_{2}^{t}<\ldots
$$

such that

$$
x_{t}\left(N_{k}^{t}\right) \rightarrow x_{t}, p_{t}\left(N_{k}^{t}\right) \rightarrow p_{t}, q_{t}\left(N_{k}^{t}\right) \rightarrow q_{t} \text { (a.s.) as } k \rightarrow \infty,
$$

and $N_{1}^{t}, N_{2}^{t}, \ldots$ is a subsequence of $N_{1}^{t-1}, N_{2}^{t-1}, \ldots$ which can be represented as

$$
N_{m}^{t}=N_{k(m)}^{t-1},
$$

where $0<k(1)<k(2)<\ldots$ are $\mathcal{F}_{t}$-measurable integer-valued random variables.

Define

$$
q_{t}(N):=E_{t} p_{t+1}(N)(0 \leq t \leq N) .
$$


Observe that for each $t=1,2, \ldots$ and $N \geq t$, we have

$$
\begin{aligned}
& \left|x_{t}(N)\right| \leq K^{t}(\text { a.s. }), E\left|p_{t}(N)\right| \leq C^{t}, E\left|q_{t}(N)\right| \leq C^{t+1}, \\
& \left(p_{t}(N), q_{t}(N)\right) \in Z_{t}^{\times} \text {(a.s.), } \\
& \left(x_{t-1}(N), x_{t}(N)\right) \in Z_{t} \text { (a.s.), } \\
& p_{t}(N) x_{t-1}(N)=1 \text { (a.s.), } \\
& q_{t-1}(N)=E_{t-1} p_{t}(N) \text { (a.s.), }
\end{aligned}
$$

where the inequalities in (44) follow from (27), (28) and (43), relations (45)-(47) hold because $p_{1}(N), \ldots, p_{N+1}(N)$ is a dual path supporting the path $x_{0}(N), \ldots, x_{N}(N)$, and (48) is valid by the definition of $q_{t-1}(N)$.

Note that relations (45)-(47) will remain valid if we replace $N \geq t$ by any random $N(\omega) \geq t$. This is also true for (48) if $N(\omega)$ is $\mathcal{F}_{t-1}$-measurable. Indeed, we have

$$
\begin{aligned}
q_{t-1}(N(\omega), \omega) & =\sum_{m} \mathbf{1}_{\{N(\omega)=m\}} q_{t-1}(m, \omega)=\sum_{m} \mathbf{1}_{\{N(\omega)=m\}} E_{t-1} p_{t}(m, \omega) \\
& =E_{t-1} \sum_{m} \mathbf{1}_{\{N(\omega)=m\}} p_{t}(m, \omega)=E_{t-1} p_{t}(N(\omega), \omega) \text { (a.s.). }
\end{aligned}
$$

Let us construct a triplet (35) and a sequence (40) satisfying for $t=1$ all the conditions (36)-(42) except for (39). We will apply Proposition A.1 (see the Appendix) to the sequence of $3 n$-dimensional $\mathcal{F}_{1}$-measurable random vectors $w_{1}(N)=\left(x_{1}(N), p_{1}(N), q_{1}(N)\right)(N \geq 1)$. For each $N=1,2, \ldots$, these vectors satisfy conditions (44) with $t=1$, which implies that $\lim \inf E\left|w_{1}(N)\right|<\infty$. By virtue of Proposition A.1, there exists a vector function $w_{1}=$ $\left(x_{1}, p_{1}, q_{1}\right)$ and a sequence of $\mathcal{F}_{1}$-measurable integer-valued functions $1<N_{1}^{1}<N_{2}^{1}<\ldots$ satisfying (35) and (41) with $t=1$. Since the sets $Z_{t}^{\times}(\omega)$ and $Z_{t}(\omega)$ are closed, the relations

$$
\begin{aligned}
& \left(p_{1}\left(N_{k}^{1}\right), q_{1}\left(N_{k}^{1}\right)\right) \in Z_{1}^{\times}, k=1,2, \ldots \text { (a.s.), } \\
& \left(x_{0}, x_{1}\left(N_{k}^{1}\right)\right) \in Z_{1}, k=1,2, \ldots \text { (a.s.), } \\
& p_{1}\left(N_{k}^{1}\right) x_{0}=1, k=1,2, \ldots \text { (a.s.) }
\end{aligned}
$$

yield in the limit (36)-(38) for $t=1$. Note that $\left\|x_{1}\right\|_{\infty}<\infty$ because $\left(x_{0}, x_{1}\right) \in Z_{1}$ (a.s.), and so $\left\|x_{1}\right\|_{\infty} \leq K_{1}\left\|x_{0}\right\|_{\infty}$.

Suppose a triplet (35) and a sequence (40) satisfying (36)-(42) are constructed for some $t \geq 1$; let us construct such a triplet and a sequence for $t+1$. Since $N_{k}^{t}(\omega) \geq t+1$, relations (45)-(48) (with $t+1$ in place of $t$ ) imply

$$
\begin{aligned}
& \left(p_{t+1}\left(N_{k}^{t}\right), q_{t+1}\left(N_{k}^{t}\right)\right) \in Z_{t+1}^{\times} \text {(a.s.), } \\
& \left(x_{t}\left(N_{k}^{t}\right), x_{t+1}\left(N_{k}^{t}\right)\right) \in Z_{t+1} \text { (a.s.), } \\
& p_{t+1}\left(N_{k}^{t}\right) x_{t}\left(N_{k}^{t}\right)=1 \text { (a.s.), } \\
& q_{t}\left(N_{k}^{t}\right)=E_{t} p_{t+1}\left(N_{k}^{t}\right) \text { (a.s.) }
\end{aligned}
$$

for all $k=1,2, \ldots$. The last equality holds because the integer-valued random variable $N_{k}^{t}$ is $\mathcal{F}_{t}$-measurable [see (49)]. By the construction of the triplet $\left(x_{t}, p_{t}, q_{t}\right)$ and the sequence $N_{k}^{t}$, we have

$$
q_{t}\left(N_{k}^{t}\right) \rightarrow q_{t} \text { (a.s.), } x_{t}\left(N_{k}^{t}\right) \rightarrow x_{t} \text { (a.s.). }
$$

By virtue of (56) and (57), we have

$$
E_{t} p_{t+1}\left(N_{k}^{t}\right)=q_{t}\left(N_{k}^{t}\right) \rightarrow q_{t}(\text { a.s. }) .
$$


We apply the conditional version of the multidimensional Fatou's lemma (see the Appendix, Proposition A.3) to the sequence of $\mathcal{F}_{t+1}$-measurable random vectors $p_{k}(\omega):=$ $p_{t+1}\left(N_{k}^{t}(\omega), \omega\right)$ whose values belong to the cone $C(\omega):=X_{t}^{*}(\omega)$ (a.s.), depending $\mathcal{F}_{t^{-}}$ measurably on $\omega$. The conditional expectations $E_{t}\left|p_{k}(\omega)\right|$ are finite (a.s.) because

$$
\begin{aligned}
E_{t}\left|p_{t+1}\left(N_{k}^{t}(\omega), \omega\right)\right| & =E_{t} \sum_{m} \mathbf{1}_{\left\{N_{k}^{t}=m\right\}}\left|p_{t+1}(m, \omega)\right| \\
& =\sum_{m} \mathbf{1}_{\left\{N_{k}^{t}=m\right\}} E_{t}\left|p_{t+1}(m, \omega)\right|<\infty \text { (a.s.) }
\end{aligned}
$$

(cf. (49)), where $E_{t}\left|p_{t+1}(m, \omega)\right|<\infty$ (a.s.) since $E\left|p_{t+1}(m, \omega)\right|<\infty$. Furthermore, $E_{t} p_{k}(\omega) \rightarrow q_{t}(\omega)$ (a.s.) by virtue of (58)]. Consequently, Proposition A.3 can be applied, and we obtain that there exists a sequence $1<k_{1}(\omega)<k_{2}(\omega)<\ldots$ of $\mathcal{F}_{t+1}$-measurable integer-valued functions and an $\mathcal{F}_{t+1}$-measurable vector function $p_{t+1}(\omega) \in C(\omega)$ (a.s.) such that

$$
\begin{aligned}
& p_{k_{l}} \rightarrow p_{t+1} \text { (a.s.) as } l \rightarrow \infty, \\
& q_{t}-E_{t} p_{t+1} \in C(\omega) \text { (a.s.). }
\end{aligned}
$$

Since $q_{t} \in \mathcal{Q}_{t}$, inequality (60) implies that $p_{t+1} \in \mathcal{P}_{t+1}$. Indeed, we have $\dot{y}_{t}(\omega) \in X_{t}(\omega)$ and $q_{t}-E_{t} p_{t+1} \in C(\omega)=X_{t}^{*}(\omega)$ (a.s.), consequently, $q_{t} \stackrel{\circ}{y}_{t}-E_{t} p_{t+1} \stackrel{\circ}{y}_{t} \geq 0$ (a.s.), which yields

$$
\left\|\stackrel{\circ}{y}_{t}\right\|_{\infty} E\left|q_{t}\right| \geq E q_{t} \stackrel{\circ}{y}_{t} \geq E E_{t} p_{t+1} \stackrel{\circ}{y}_{t}=E p_{t+1} \stackrel{\circ}{y}_{t} \geq \frac{\varepsilon_{t}}{m_{t}} E\left|p_{t+1}\right| .
$$

The last inequality follows from Lemma 1 because $p_{t+1}(\omega) \in C(\omega)=X_{t}^{*}(\omega)$ (a.s.) and $\mathbb{B}\left(\dot{\circ}_{t}, \varepsilon_{t}\right) \subseteq X_{t}(\omega)$ (a.s.).

By setting $n_{l}^{t+1}:=N_{k_{l}}^{t}$, we obtain a sequence

$$
t+1<n_{1}^{t+1}<n_{2}^{t+1}<\ldots
$$

of $\mathcal{F}_{t+1}$-measurable integer-valued functions such that

$$
p_{t+1}\left(n_{l}^{t+1}\right) \rightarrow p_{t+1}, x_{t}\left(n_{l}^{t+1}\right) \rightarrow x_{t}(\text { a.s. }) \text { as } l \rightarrow \infty
$$

by virtue of (59) and (57). Note that the first inequality in (61) holds because $n_{1}^{t+1}=N_{k_{1}}^{t}>$ $N_{1}^{t}>1$. In view of (53)-(55), we get

$$
\begin{array}{r}
\left(p_{t+1}\left(n_{l}^{t+1}\right), q_{t+1}\left(n_{l}^{t+1}\right) \in Z_{t+1}^{\times}\right. \text {(a.s.) } \\
\left(x_{t}\left(n_{l}^{t+1}\right), x_{t+1}\left(n_{l}^{t+1}\right) \in Z_{t+1}\right. \text { (a.s.) } \\
p_{t+1}\left(n_{l}^{t+1}\right) x_{t}\left(n_{l}^{t+1}\right)=1 \text { (a.s.). }
\end{array}
$$

Since the sequence $p(l):=p_{t+1}\left(n_{l}^{t+1}\right)$ converges for almost all $\omega$, it is bounded for almost all $\omega$. By virtue of (63) and Lemma 3, the sequence $q(l):=q_{t+1}\left(n_{l}^{t+1}\right)$ is a.s. bounded too. Lemma 3 can be applied because by definition, $q_{t+1}(N)=E_{t+1} p_{t+2}(N)$, and so $q_{t+1}(N) \in X_{t+1}^{*}$ (a.s.) [recall that $p_{t+2}(N) \in X_{t+1}^{*}$ (a.s.)].

For $x(l):=x_{t+1}\left(n_{l}^{t+1}\right)$, we have $|x(l)| \leq K^{t+1}$ (a.s.) according to (27). Therefore we can apply Proposition A.1 to the sequence of $\mathcal{F}_{t+1}$-measurable random vectors $v_{l}:=$ $(x(l), q(l))$. By virtue of this proposition, there exists a sequence $1<l(1)<l(2)<\ldots$ 
of $\mathcal{F}_{t+1}$-measurable integer-valued random variables and $\mathcal{F}_{t+1}$-measurable random vectors $x_{t+1} \in X_{t+1}$ (a.s.) and $q_{t+1} \in X_{t+1}^{*}$ (a.s.) for which

$$
x(l(m))=x_{t+1}\left(n_{l(m)}^{t+1}\right) \rightarrow x_{t+1}, q(l(m))=q_{t+1}\left(n_{l(m)}^{t+1}\right) \rightarrow q_{t+1} \text { (a.s.). }
$$

Since the sets $Z_{t+1}^{\times}(\omega)$ and $Z_{t+1}(\omega)$ are closed, it follows from (63)-(66) and (62) that

$$
\begin{aligned}
& \left(p_{t+1}, q_{t+1}\right) \in Z_{t+1}^{\times} \text {(a.s.), } \\
& \left(x_{t}, x_{t+1}\right) \in Z_{t+1} \text { (a.s.), } \\
& p_{t+1} x_{t}=1 \text { (a.s.). }
\end{aligned}
$$

We have $\left|x_{t+1}\right| \leq K^{t+1}$ (a.s.) because $|x(l)| \leq K^{t+1}$ (a.s.). Since $q_{t+1} \in X_{t+1}^{*}$ (a.s.) and $\left(p_{t+1}, q_{t+1}\right) \in Z_{t+1}^{\times}$(a.s.), by Lemma 3 we get $\left|q_{t+1}\right| \leq C_{t+1}\left|p_{t+1}\right|$, and so $q_{t+1} \in \mathcal{Q}_{t+1}$ as long as $p_{t+1} \in \mathcal{P}_{t+1}$, which was shown above. Thus the triplet $\left(x_{t+1}, p_{t+1}, q_{t+1}\right) \in$ $\mathcal{X}_{t+1} \times \mathcal{P}_{t+1} \times \mathcal{Q}_{t+1}$ satisfies all the conditions listed in (36)-(39) (with $t+1$ in place of $t$ ).

It remains to define the sequence $N_{m}^{t+1}, m=1,2, \ldots$, of $\mathcal{F}_{t+1}$-measurable random integers by

$$
N_{m}^{t+1}:=n_{l(m)}^{t+1}=N_{k(l(m))}^{t}\left[n_{l}^{t+1}:=N_{k(l)}^{t}\right] .
$$

The sequence $N_{m}^{t+1}$ is strictly increasing in $m$ because the sequences $N_{k}^{t}, k(l), n_{l}^{t+1}$ and $l(m)$ are strictly increasing. We have $N_{1}^{t+1}>t+1$ since $N_{1}^{t}>t$ and $k(l)>1$. By using formulas (66) and (62), we obtain

$$
x_{t+1}\left(N_{m}^{t+1}\right) \rightarrow x_{t+1}, p_{t+1}\left(N_{m}^{t+1}\right) \rightarrow p_{t+1}, q_{t+1}\left(N_{m}^{t+1}\right) \rightarrow q_{t+1} \text { (a.s.). }
$$

thus the sequence $N_{m}^{t+1}$ possesses all the properties required for $t+1$ in (41) and (42) [the latter follows from (70)].

We have constructed for each $t=1,2, \ldots$, vector functions $x_{t}(\omega), p_{t}(\omega), q_{t}(\omega)$ satisfying (35)-(39). Consider the sequences $x_{0}, x_{1}, \ldots$ and $p_{1}, p_{2}, \ldots$ It follows from (37) that the former is a path. Let us show that the latter is a dual path. The inequalities (39) (with $t+1$ in place of $t$ ) and (36) imply that there exists a vector function $h_{t} \in \mathcal{Q}_{t}$ such that for almost all $\omega$,

$$
q_{t}(\omega)=\left(E_{t} p_{t+1}\right)(\omega)+h_{t}(\omega), h_{t}(\omega) \in X_{t}^{*}(\omega),
$$

and $\left(p_{t}(\omega), q_{t}(\omega)\right) \in Z_{t}^{\times}(\omega)$. The last inclusion means that $q_{t}(\omega) b \leq p_{t}(\omega) a$ for all $(a, b) \in$ $Z_{t}(\omega)$. Taking into account (71), we get

$$
b\left(E_{t} p_{t+1}\right)(\omega)+b h_{t}(\omega) \leq a p_{t}(\omega), \quad(a, b) \in Z_{t}(\omega) .
$$

Since $b \in X_{t}(\omega)$ as long as $(a, b) \in Z_{t}(\omega)$, we obtain that $b h_{t}(\omega) \geq 0$ [see (71)]. Consequently, we get

$$
b\left(E_{t} p_{t+1}\right)(\omega) \leq a p_{t}(\omega), \quad(a, b) \in Z_{t}(\omega),
$$

i.e. $\left(p_{t}(\omega),\left(E_{t} p_{t+1}\right)(\omega)\right) \in Z_{t}^{\times}(\omega)$, which proves that $p_{1}(\omega), p_{2}(\omega), \ldots$ is a dual path. It remains to observe that $p_{1}, p_{2}, \ldots$ supports $x_{0}, x_{1}, \ldots$ by virtue of (38).

\section{Growth-optimality of rapid paths}

Proof of Theorem 3 Let $x_{0}, x_{1}, \ldots$ be a rapid path supported by a dual path $p_{1}, p_{2}, \ldots$ Let us first observe that for any path $\left(y_{t}\right)$ the sequence $p_{t+1} y_{t}(t=1,2, \ldots)$ is a non-negative 
supermartingale with respect to the filtration $\mathcal{F}_{1} \subseteq \mathcal{F}_{2} \subseteq \ldots$. This is immediate from the relations:

$$
E_{t} p_{t+1} x_{t}=\bar{p}_{t+1} x_{t} \leq p_{t} x_{t-1} \text { (a.s.), } t=1,2, \ldots
$$

following from (9).

Further, consider any $y \in L_{\infty}\left(\mathcal{F}_{t+l}, \mathbb{R}^{m_{t+l}}\right)$ with $|y(\omega)| \leq \gamma\left|y_{t}(\omega)\right|$. By using (9) and (23), we have

$$
p_{t+l} y_{t+l-1} \geq \bar{p}_{t+l+1}\left(y_{t+l}+y\right)=\bar{p}_{t+l+1} y_{t+l}+\bar{p}_{t+l+1} y \geq \bar{p}_{t+l+1} y \text { (a.s.) }
$$

because $\bar{p}_{t+l+1} y_{t+l} \geq 0$ (a.s.). The last inequality is valid since $y_{t+l}(\omega) \in X_{t+l}(\omega)$ and $p_{t+l+1}(\omega) \in X_{t+l}^{*}(\omega)$ (a.s.), which yields $p_{t+l+1} y_{t+l} \geq 0$ (a.s.) and so $\bar{p}_{t+l+1} y_{t+l} \geq 0$ (a.s.). Put

$$
y=\frac{\bar{p}_{t+l+1}}{\left|\bar{p}_{t+l+1}\right|} \gamma\left|y_{t}\right| \text {. }
$$

Then $|y(\omega)|=\gamma\left|y_{t}(\omega)\right|$ and $y \in L_{\infty}\left(\mathcal{F}_{t+l}, \mathbb{R}^{m_{t+l}}\right)$. Consequently, (73) can be applied to $y$ defined by (74). Observe that

$$
\bar{p}_{t+l+1} y=\frac{\|\left.\bar{p}_{t+l+1}\right|^{2}}{\left|\bar{p}_{t+l+1}\right|} \gamma\left|y_{t}\right| \geq\left|\bar{p}_{t+l+1}\right|\left(m_{t+l}\right)^{-1} \gamma\left|y_{t}\right|,
$$

where $\|\cdot\|$ is the Euclidean norm in $\mathbb{R}^{m_{t+l}}$ (we use the inequality $\|\cdot\| \geq|\cdot| / \sqrt{m_{t+l}}$ ). Further, the equality $p_{t+l+1} x_{t+l}=1$ implies $\bar{p}_{t+l+1} x_{t+l}=1$, and so

$$
\left|\bar{p}_{t+l+1}\right|\left|x_{t+l}\right| \geq 1 \text {, }
$$

and it follows from (A3) with a constant $K_{t}=K$ independent of $t$ that

$$
\left|x_{t+l}\right| \leq K^{l}\left|x_{t}\right|
$$

By combining (76) and (77), we get

$$
\left|\bar{p}_{t+l+1}\right| \geq K^{-l}\left|x_{t}\right|^{-1}
$$

and by using (75), (73) and (78), we obtain

$$
p_{t+l} y_{t+l-1} \geq K^{-l}\left(m_{t+l}\right)^{-1} \gamma\left|y_{t}\right|\left|x_{t}\right|^{-1} \geq K^{-l} m^{-1} \gamma\left|y_{t}\right|\left|x_{t}\right|^{-1} \text { (a.s.), }
$$

which yields

$$
p_{t+1} y_{t} \geq E_{t+1} p_{t+l} y_{t+l-1} \geq K^{-l} m^{-1} \gamma\left|y_{t}\right|\left|x_{t}\right|^{-1} \text { (a.s.). }
$$

Since $p_{t+1} y_{t}$ is a non-negative supermartingale, the proof is complete.

\section{A financial market model}

In this section we consider a model for a financial market with transaction costs and portfolio constraints in which the cones $X_{t}(\omega)$ and $Z_{t}(\omega)$ are polyhedral. We check conditions (A1)(A5) guaranteeing that Theorems 1,2, and 3 can be applied to the model. Additionally, we verify assumption $(\mathbf{L})$ and the following conditions $(\mathbf{F})$ and $\left(\mathrm{A}^{\prime}\right)$ that will be used further in our work. 
(F) There exist $\mathcal{F}_{t}$-measurable random vectors $f_{t, p}(\omega), p=1, \ldots, P$, such that for each $\omega$ we have $f_{t, p}(\omega) \neq 0$,

$$
X_{t}(\omega)=\left\{a: a=\sum_{p=1}^{P} f_{t, p}(\omega) c^{p} \text { for some } c^{p} \geq 0, p=1, \ldots, P\right\} .
$$

and

$$
\theta_{t}|c| \leq\left|\sum_{p=1}^{P} f_{t, p}(\omega) c^{p}\right| \leq \Theta_{t}|c|, c=\left(c^{1}, \ldots, c^{P}\right) \in \mathbb{R}_{+}^{P},
$$

where $0<\theta_{t}<\Theta_{t}(t=0,1, \ldots)$ are constants and $P$ is a natural number.

$\left(A 4^{\prime}\right)$ For every $t \geq 1$ there exist a strictly positive constant $\alpha_{t}>0$ and a bounded vector function $\hat{z}_{t}(\omega)=\left(\hat{x}_{t-1}(\omega), \hat{y}_{t}(\omega)\right)$ such that $\hat{x}_{t-1}(\omega)$ is $\mathcal{F}_{t-1}$-measurable, $\hat{y}_{t}(\omega)$ is $\mathcal{F}_{t}$-measurable and $\mathbb{B}\left(\hat{z}_{t}(\omega), \alpha_{t}\right) \subseteq Z_{t}(\omega)$ for all $\omega$.

Clearly $\left(A 4^{\prime}\right)$ implies (A4).

We consider a market where $m$ assets are traded at dates $t=1,2, \ldots$ Random vectors $a(\omega) \in \mathbb{R}^{m}$ are interpreted as (contingent) portfolios of assets. Positions $a^{i}(\omega)$ of the portfolio $a(\omega)=\left(a^{1}(\omega), \ldots, a^{m}(\omega)\right) \in \mathbb{R}^{m}$ are measured in terms of their values in the market prices. We omit $\omega$ in the notation where it does not lead to ambiguity.

For each $t=0,1, \ldots$ and $i=1, \ldots, m$ the following $\mathcal{F}_{t}$-measurable random variables are given: margin requirement coefficients for long and short positions $0<\mu_{t, i}^{+}<\mu_{t, i}^{-}$, market asset prices $S_{t, i}>0$, transaction cost rates for selling and buying assets $0 \leq \lambda_{t, i}^{+}<1$, $\lambda_{t, i}^{-} \geq 0$, dividend or interest yield rates for long and short positions $0 \leq D_{t, i}^{+} \leq D_{t, i}^{-}$. We denote by $R_{t, i}=S_{t, i} / S_{t-1, i}$ the return on asset $i$.

Portfolio constraints in the model are specified by the cones

$$
X_{t}(\omega)=\left\{a \in \mathbb{R}^{m}: \sum_{i=1}^{m} \mu_{t, i}^{+}(\omega) a_{+}^{i} \geq \sum_{i=1}^{m} \mu_{t, i}^{-}(\omega) a_{-}^{i}\right\}
$$

where $a_{-}=(-a)_{+}$. The random variables $\mu_{t, i}^{ \pm}$can be used to define margin requirements as in the following two particular cases of (83):

$$
\begin{aligned}
& X_{t}(\omega)=\left\{a \in \mathbb{R}^{m}:\left|a_{+}\right| \geq U_{t}\left|a_{-}\right|\right\}, \text {or } \\
& X_{t}(\omega)=\left\{a \in \mathbb{R}^{m}: \sum_{i=1}^{m}\left(1-\lambda_{t, i}^{+}(\omega)\right) a_{+}^{i} \geq U_{t} \sum_{i=1}^{m}\left(1+\lambda_{t, i}^{-}(\omega)\right) a_{-}^{i}\right\},
\end{aligned}
$$

where $U_{t}>1$ are constants. In both (84) and (85), $U_{t}$ can be interpreted as a margin requirement coefficient: a trader must be able to liquidate the long positions of her portfolio to cover the short positions with excess determined by $U_{t}$. In (84) no transaction costs are taken into account in the liquidation value. In (85), there are proportional transaction costs specified in terms of $\lambda_{t, i}^{ \pm}$.

Trading in the market under consideration proceeds as follows. At each date $t$, the trader receives the dividend on her portfolio $a(\omega)$ purchased at the previous date. The amount of dividend is specified by the function $d_{t}(\omega, a)$ defined by the formula

$$
d_{t}(a)=\sum_{i=1}^{m}\left(D_{t, i}^{+} a_{+}^{i}-D_{t, i}^{-} a_{-}^{i}\right)
$$


Here $D_{t, i}^{ \pm}$specify the amount of dividend received or returned for each unit of cash invested in asset $i$. (We assume that dividends on short positions must be returned. The dividend rates for long and short positions might be different due to the presence of taxes on dividends). The dividends received or returned for a physical unit of asset $i$ are $D_{t, i}^{ \pm} S_{t-1, i}$.

After that, the trader rearranges her portfolio $a(\omega)$ with added dividend to a portfolio $b(\omega)$ subject to the self-financing constraint $\psi_{t}(\omega, a, b) \geq 0$, where

$$
\psi_{t}(a, b)=\sum_{i=1}^{m}\left(1-\lambda_{t, i}^{+}\right)\left(R_{t, i} a^{i}-b^{i}\right)_{+}-\sum_{i=1}^{m}\left(1+\lambda_{t, i}^{-}\right)\left(R_{t, i} a^{i}-b^{i}\right)_{-}+d_{t}(a) .
$$

The first sum represents the amount of money the trader receives by selling assets, the second sum is the amount of money she pays for buying assets, including transaction costs.

The above description of the model corresponds to the cones

$$
Z_{t}(\omega):=\left\{(a, b) \in X_{t-1}(\omega) \times X_{t}(\omega): \psi_{t}(\omega, a, b) \geq 0\right\} .
$$

As a liquidation value function, which appears in condition $(\mathbf{L})$, we can use

$$
\phi_{t}(\omega, b)=\sum_{i=1}^{m}\left(1-\lambda_{t, i}^{+}(\omega)\right) b_{+}^{i}-\sum_{i=1}^{m}\left(1+\lambda_{t, i}^{-}(\omega)\right) b_{-}^{i} .
$$

Conditions under which the function (87) satisfies (L) are given in Proposition 2 below.

A natural instance of this model is when asset 1 represents cash deposited with a bank account and the other assets are shares of stock. Then $S_{t, 1}=1$ and $\lambda_{t, 1}^{ \pm}=0$ (the value is expressed in terms of cash and there are no transaction costs for cash). The random variables $D_{t, 1}^{ \pm}$are interest rates for lending and borrowing and the random variables $D_{t, i}^{ \pm}, i \geq 2$, are dividend yield rates on stock. Different dividend yield rates for long and short positions correspond to the situation when some assets pay dividends in a currency different from asset 1 and there is a bid-ask spread in the exchange rates.

Observe that $Z_{t}(\omega)$ is indeed a cone: clearly it contains with any vector $(a, b)$ all vectors $\lambda(a, b)$, where $\lambda \geq 0$. Also it is convex, since the function $\psi_{t}(a, b)$ is concave as follows from the representation

$$
\begin{aligned}
\psi_{t}(a, b)= & \sum_{i=1}^{m}\left[\left(1-\lambda_{t, i}^{+}\right)\left(R_{t, i} a^{i}-b^{i}\right)+D_{t, i}^{+} a^{i}\right] \\
& -\sum_{i=1}^{m}\left[\left(\lambda_{t, i}^{-}+\lambda_{t, i}^{+}\right)\left(R_{t, i} a^{i}-b^{i}\right)_{-}+\left(D_{t, i}^{-}-D_{t, i}^{+}\right) a_{-}^{i}\right],
\end{aligned}
$$

where the first sum is a linear function of $a, b$ and the second sum is a convex function of $a, b$.

We introduce the following assumptions. To shorten the notation, we put $\Lambda_{t, i}^{+}=1-\lambda_{t, i}^{+}$ and $\Lambda_{t, i}^{-}=1+\lambda_{t, i}^{-}$.

(B1) For each $t$, there exist constants $\underline{R}_{t}, \bar{R}_{t}, \underline{\Lambda}_{t}, \bar{\Lambda}_{t}, \bar{D}_{t}$ such that $0<\underline{R}_{t} \leq R_{t, i}(\omega) \leq \bar{R}_{t}$, $0<\underline{\Lambda}_{t} \leq \Lambda_{t, i}^{+}(\omega), \Lambda_{t, i}^{-}(\omega) \leq \bar{\Lambda}_{t}, D_{t, i}^{-}(\omega) \leq \bar{D}_{t}$ for all $i, \omega$.

(B2) For each $t$, there exists a constant $\mu_{t}$ such that $\mu_{t, i}^{-}(\omega) / \mu_{t, j}^{+}(\omega) \geq \mu_{t}$ for all $\omega, i \neq j$, and $\mu_{t}>v_{t}$, where $v_{t}:=\max \left\{\left(\bar{\Lambda}_{t+1} \bar{R}_{t+1}+\bar{D}_{t+1}\right) /\left(\underline{\Lambda}_{t+1} \underline{R}_{t+1}+\underline{D}_{t+1}\right) ; \bar{\Lambda}_{t} / \underline{\Lambda}_{t}\right\}$ and $\underline{D}_{t} \geq 0$ is a constant such that $\underline{D}_{t} \leq D_{t, i}^{+}(\omega)$ for all $\omega, i$.

Observe that for the particular examples of the cones $X_{t}(\omega)$ in (84) and (85), if condition (B1) is satisfied then (B2) will hold if $U_{t}>v_{t}$ for each $t$. 
Proposition 1 Let conditions (B1) and (B2) hold. Then the cones $X_{t}(\omega)$ satisfy conditions $(\mathbf{F})$ and $(\mathrm{A} 1)$ and the cones $Z_{t}(\omega)$ satisfy conditions (A2), (A3) and (A4').

To prove Proposition 1 we will need the following auxiliary result .

Lemma 1 Let conditions (B1), (B2) hold. Then

(a) For each $t$ there exists a constant $C_{t}^{1}>0$ such that if $a \in X_{t}(\omega)$ then $\left|a_{+}\right|-v_{t}\left|a_{-}\right| \geq$ $C_{t}^{1}|a|$.

(b) For each t there exists a constant $C_{t}^{2}$ such that if $a \in X_{t-1}(\omega), b \in X_{t}(\omega)$ and $|b| \leq$ $C_{t}^{2}|a|$, then $(a, b) \in Z_{t}(\omega)$.

Proof (a) Consider the non-random cone $\tilde{X}_{t}=\left\{a \in \mathbb{R}^{m}: \mu_{t}\left|a_{-}\right| \leq\left|a_{+}\right|\right\}$. Condition (B2) implies that $X_{t}(\omega) \subseteq \tilde{X}_{t}$. Observe that since $\mu_{t}>1$ we have $\tilde{X}_{t} \cap\left(-\tilde{X}_{t}\right)=\{0\}$. The continuous function $h_{t}(a)=\left|a_{+}\right|-v_{t}\left|a_{-}\right|$is strictly positive on the compact set $\hat{X}_{t}=\tilde{X}_{t} \cap\{a:|a|=1\}$. Indeed, since $h_{t}(a) \geq\left(\mu_{t}-v_{t}\right)\left|a_{-}\right|$on $\tilde{X}_{t}$, then the equality $h_{t}(a)=0$ would imply $\left|a_{-}\right|=0$, and hence $\left|a_{+}\right|=h_{t}(a)=0$, so that $|a|=0$. Then $h_{t}(a)$ attains a strictly positive minimum on $\hat{X}_{t}$, which can be taken as $C_{t}^{1}$.

(b) Let $b \in X_{t}(\omega)$. It is straightforward to check that for any numbers $x, y$ we have $(x-$ $y)_{+} \geq x_{+}-y_{+}$and $(x-y)_{-} \leq x_{-}+y_{+}$. Using this, we obtain for any $a \in X_{t-1}(\omega)$

$$
\begin{aligned}
\psi_{t}(a, b) & \geq \sum_{i}\left(\left(\Lambda_{t, i}^{+} R_{t, i}+D_{t, i}^{+}\right) a_{+}^{i}-\left(\Lambda_{t, i}^{-} R_{t, i}+D_{t, i}^{-}\right) a_{-}^{i}\right)-\sum_{i}\left(\Lambda_{t, i}^{+}+\Lambda_{t, i}^{-}\right) b_{+}^{i} \\
& \geq\left(\underline{\Lambda}_{t} \underline{R}_{t}+\underline{D}_{t}\right)\left|a_{+}\right|-\left(\bar{\Lambda}_{t} \bar{R}_{t}+\bar{D}_{t}\right)\left|a_{-}\right|-2 \bar{\Lambda}_{t}\left|b_{+}\right| \\
& \geq\left(\underline{\Lambda}_{t} \underline{R}_{t}+\underline{D}_{t}\right)\left(\left|a_{+}\right|-v_{t-1}\left|a_{-}\right|\right)-2 \bar{\Lambda}_{t}|b| \\
& \geq C_{t-1}^{1}\left(\underline{\Lambda}_{t} \underline{R}_{t}+\underline{D}_{t}\right)|a|-2 \bar{\Lambda}_{t}|b| .
\end{aligned}
$$

Then statement (b) can be fulfilled with the constant $C_{t}^{2}=C_{t-1}^{1}\left(\underline{\Lambda}_{t} \underline{R}_{t}+\underline{D}_{t}\right) /\left(2 \bar{\Lambda}_{t}\right)$, since in that case $\psi_{t}(a, b) \geq 0$, implying $(a, b) \in Z_{t}$.

Proof of Proposition 1 Let us observe that the cones $X_{t}$ satisfy $(\mathbf{F})$. We first show that each cone $X_{t}$ is polyhedral. Put $f_{t, i, j}=e_{i}-\left(\mu_{t, i}^{+} / \mu_{t, j}^{-}\right) e_{j}$ for $i \neq j$, where $e_{i}$ is the $i$-th basis vector in $\mathbb{R}^{m}$. Suppose $a \in X_{t}(\omega), a \neq 0$. Denote by $I=\left\{i: a^{i}>0\right\}, J=\left\{j: a^{j}<0\right\}$ the sets of indices of positive and negative coordinates of $a$ and put $\delta=\left(\sum_{j \in J} \mu_{t, j}^{-}\left|a^{j}\right|\right) /\left(\sum_{i \in I} \mu_{t, i}^{+} a^{i}\right)$. Clearly, $\delta \leq 1$ as $a \in X_{t}$. Then

$$
a=\delta \sum_{i \in I} \sum_{j \in J} \frac{a^{i} \mu_{t, j}^{-}\left|a^{j}\right|}{\sum_{k \in J} \mu_{t, k}^{-}\left|a^{k}\right|} f_{t, i, j}+(1-\delta) \sum_{i \in I} a^{i} e_{i} .
$$

Hence the cone $X_{t}$ can be represented in the form (81) with $m^{2}$ generators: $f_{t, i, j}$ and $e_{i}$ for $i, j=1, \ldots, m, j \neq i$.

Since $f_{t, p}(\omega) \neq 0$, we can assume without loss of generality that all the generators $f_{t, p}(\omega)$ of the cone $X_{t}(\omega)$ are normalized: $\left|f_{t, p}(\omega)\right|=1$. Then the second inequality in (82) will hold with $\Theta_{t}=1$.

To prove the first inequality in (82), observe that the minimum of the continuous function $v\left(c, f_{1}, \ldots, f_{P}\right):=\left|\sum_{p} c_{p} f_{p}\right|$ is strictly positive on the compact set $\left\{(c, f): c \in \mathbb{R}_{+}^{P},|c|=\right.$ $\left.1, f_{p} \in \tilde{X}_{t},\left|f_{p}\right|=1, p=1, \ldots, P\right\}$. Then $\theta_{t}$ can be taken equal to this minimum. 
Let us check condition (A1). In the proof of Lemma 1, we observed that the cones $X_{t}(\omega)$ is contained in the non-random cone $\tilde{X}_{t}=\left\{a \in \mathbb{R}^{m}: \mu\left|a_{-}\right| \leq\left|a_{+}\right|\right\}$. Put $q_{t}=e$, where $e=(1, \ldots, 1) \in \mathbb{R}^{m}$. Indeed, $q_{t} \in X_{t}^{*}(\omega)$ since for any $a=\left(a^{1}, \ldots, a^{m}\right) \in X_{t}(\omega)$

$$
q_{t}(\omega) a=\sum_{i=1}^{m} a^{i}=\left|a_{+}\right|-\left|a_{-}\right| \geq\left(\mu_{t}-1\right)\left|a_{-}\right| \geq 0 .
$$

Note that the continuous function $q_{t}(a)=\sum_{i=1}^{m} a^{i}$ is strictly positive on the compact set $\hat{X}_{t}=\tilde{X}_{t} \cap\{a:|a|=1\}$. Indeed, since $q_{t}(a) \geq\left(\mu_{t}-1\right)\left|a_{-}\right|$on $\tilde{X}_{t}$, the equality $q_{t}(a)=0$ would imply $|a|=0$. Then $q_{t}(a)$ attains a strictly positive minimum $Q_{t} \leq 1$ on $\hat{X}_{t}$. Define $H_{t}=Q_{t}^{-1}$. Therefore, for any $a \in X_{t}(\omega)$

$$
H_{t}^{-1}|a| \leq q_{t}(\omega) a \leq H_{t}|a|,
$$

which implies that assumption (A1) is satisfied.

Condition (A2) follows from statement (b) of Lemma 1 since for any $a \in X_{t-1}(\omega)$, $0 \leq C_{t}^{2}|a|$ and so $(a, 0) \in Z_{t}(\omega)$.

Let us prove (A3). Suppose $(a, b) \in Z_{t}$. Since for any numbers $x, y$ we have $(x-y)_{+} \leq$ $x_{+}+y_{-}$and $(x-y)_{-} \geq y_{+}-x_{+}$, we obtain

$$
\begin{aligned}
0 & \leq \psi_{t}(a, b) \leq \sum_{i}\left(\left(\Lambda_{t, i}^{+}+\Lambda_{t, i}^{-}\right) R_{t, i}+D_{t, i}^{+}\right) a_{+}^{i}+\sum_{i}\left(\Lambda_{t, i}^{+} b_{-}^{i}-\Lambda_{t, i}^{-} b_{+}^{i}\right) \\
& \leq\left(2 \bar{\Lambda}_{t} \bar{R}_{t}+\bar{D}_{t}\right)|a|+\bar{\Lambda}_{t}\left|b_{-}\right|-\underline{\Lambda}_{t}\left|b_{+}\right| \leq\left(2 \bar{\Lambda}_{t} \bar{R}_{t}+\bar{D}_{t}\right)|a|-C_{t}^{1} \underline{\Lambda}_{t}|b|,
\end{aligned}
$$

where in the last inequality we used that $b \in X_{t}(\omega)$ and according to statement (a) of Lemma 1, we have $\underline{\Lambda}_{t}\left|b_{+}\right|-\bar{\Lambda}_{t}\left|b_{-}\right| \geq \underline{\Lambda}_{t}\left(\left|b_{+}\right|-v_{t}\left|b_{-}\right|\right) \geq C_{t}^{1} \underline{\Lambda}_{t}|b|$. This implies the validity of (A3) with the constant $K_{t}=\left(2 \bar{\Lambda}_{t} \bar{R}_{t}+\bar{D}_{t}\right) /\left(C_{t}^{1} \underline{\Lambda}_{t}\right)$.

Now we will prove condition $\left(A 4^{\prime}\right)$. Let $\hat{x}=(1, \ldots, 1) \in \mathbb{R}^{m}$. Put $\hat{z}_{t}=\left(\hat{x}, \hat{y}_{t}\right)$ with $\hat{y}_{t}=\left(C_{t}^{2} / 2\right) \hat{x}$. Observe that there exists $\delta_{t}>0$ such that $\mathbb{B}\left(\hat{z}_{t}, \delta_{t}\right) \subset \mathbb{R}_{+}^{2 m}$ and therefore $\mathbb{B}\left(\hat{z}_{t}, \delta_{t}\right) \subset X_{t-1} \times X_{t}$. Since $\left|\hat{y}_{t}\right|<C_{t}^{2}|\hat{x}|$, then one can find $0<\alpha_{t} \leq \delta_{t}$ such that $\left|y_{t}\right| \leq C_{t}^{2}\left|x_{t}\right|$ for any $z_{t}=\left(x_{t-1}, y_{t}\right) \in \mathbb{B}\left(\hat{z}_{t}, \alpha_{t}\right)$. Then statement (b) of Lemma 1 implies $z_{t} \in Z_{t}$ for such $z_{t}$. Hence, the pair $\left(\hat{z}_{t}, \alpha_{t}\right)$ satisfies condition $\left(A 4^{\prime}\right)$. Therefore all conditions (A1)-(A4) are satisfied and then Theorems 1 and 2 hold.

Let us assume that assumptions (B1) and (B2) hold with constants $\mu_{t}, \underline{R}_{t}, \bar{R}_{t}, \underline{\Lambda}_{t}, \bar{\Lambda}_{t}, \bar{D}_{t}$ not depending on $t$. Under these assumptions the following assertion is valid, guaranteeing that Theorem 3 holds in the model at hand.

Proposition 2 Let assumptions (B1) and (B2) hold with constants $\mu_{t}, \underline{R}_{t}, \bar{R}_{t}, \underline{\Lambda}_{t}, \bar{\Lambda}_{t}, \bar{D}_{t}$ not depending on $t$. Then

(a) $Z_{t}(\omega)$ satisfy condition (A3) with constant $K$ not depending on $t$ and condition (A5) with $l=1$,

(b) the function $\phi_{t}(\omega, b)$ defined in (87) satisfies condition $(\boldsymbol{L})$.

Proof (a) From the proof of statement (a) of Lemma 1 one can see that if the constants from condition (B1) do not depend on $t$, then it is possible to choose $C_{t}^{1}$ independent of $t$. Then (88) implies that $K_{t}$ can be chosen independent of $t$.

Let us prove that (A5) holds. It follows from the proof of Lemma 1 that the constant $C_{t}^{2}$ can be chosen independent of $t$. Let $\gamma=C^{2} /(m+1)$ and consider any $y_{t} \in \mathcal{X}_{t}$. Put $y_{t+1}=\gamma\left|y_{t}\right| \hat{x}$, where $\hat{x}=(1, \ldots, 1) \in \mathbb{R}^{m}$. Then $\mathbb{B}\left(y_{t+1}, \gamma\left|y_{t}\right|\right) \subseteq \mathbb{R}_{+}^{m} \subseteq X_{t+1}$. Hence for any $y \in L_{\infty}\left(\mathcal{F}_{t+1}, \mathbb{R}^{m}\right)$ such that $|y| \leq \gamma\left|y_{t}\right|$ we have $y_{t+1}+y \in X_{t+1}$ and 
$\left|y_{t+1}+y\right| \leq C^{2}\left|y_{t}\right|$. Hence, statement (b) of Lemma 1 implies that $\left(y_{t}, y_{t+1}+y\right) \in Z_{t+1}$, so condition (A5) holds with $l=1$.

(b) Finally, we prove that the function $\phi_{t}(\omega, b)$ satisfies condition $(\mathbf{L})$. The second inequality in condition $(\mathbf{L})$ holds with $S=1$. Let us prove the first inequality. Since $X_{t}(\omega) \subseteq \tilde{X}_{t}=$ $\left\{a \in \mathbb{R}^{m}: \mu\left|a_{-}\right| \leq\left|a_{+}\right|\right\}$, for every $b \in X_{t}(\omega)$ we have

$$
\phi_{t}(\omega, b) \geq \underline{\Lambda}\left|b_{+}\right|-\bar{\Lambda}\left|b_{-}\right| \geq(\underline{\Lambda}-\bar{\Lambda} / \mu)\left|b_{+}\right| .
$$

Condition (B2) implies that $\mu \underline{\Lambda}>\bar{\Lambda}$. Using that and $\left|b_{+}\right| \geq\left|b_{-}\right|$, the above inequality yields $\phi_{t}(\omega, b) \geq s|b|$ with constant $s=(\underline{\Lambda}-\bar{\Lambda} / \mu) / 2>0$.

Open Access This article is licensed under a Creative Commons Attribution 4.0 International License, which permits use, sharing, adaptation, distribution and reproduction in any medium or format, as long as you give appropriate credit to the original author(s) and the source, provide a link to the Creative Commons licence, and indicate if changes were made. The images or other third party material in this article are included in the article's Creative Commons licence, unless indicated otherwise in a credit line to the material. If material is not included in the article's Creative Commons licence and your intended use is not permitted by statutory regulation or exceeds the permitted use, you will need to obtain permission directly from the copyright holder. To view a copy of this licence, visit http://creativecommons.org/licenses/by/4.0/.

\section{Appendix}

Let $(\Omega, \mathcal{F}, P)$ be a probability space and $w_{N}(\omega)(\omega \in \Omega, N=1,2, \ldots)$ a sequence of random vectors in $\mathbb{R}^{n}$.

Proposition A.1 If $\liminf \left|w_{N}(\omega)\right|<\infty$ a.s. (which is so, in particular, when $\left.\lim \inf E\left|w_{N}(\omega)\right|<\infty\right)$, then there exists a sequence of integer-valued random variables $N_{1}(\omega)<N_{2}(\omega)<\ldots$ and a random vector $w(\omega)$ such that

$$
\lim w_{N_{k}(\omega)}(\omega)=w(\omega)(\text { a.s. })
$$

and

$$
E|w(\omega)| \leq \lim \inf E\left|w_{N}(\omega)\right|
$$

Proof Define $\xi(\omega)=\lim \inf \left|w_{N}(\omega)\right|$. Since $\xi(\omega)<\infty$ (a.s.), for almost all $\omega$ there exists a sequence $v=\left(N_{k}\right)_{k=1}^{\infty}$ of natural numbers $N_{k}$ and a vector $w \in \mathbb{R}^{n}$ such that

$$
N_{k}<N_{k+1}, \lim \left|w_{N_{k}}(\omega)\right| \rightarrow \xi(\omega), \lim w_{N_{k}}(\omega)=w,|w|=\xi(\omega),
$$

Denote by $A$ the set of $(\omega, v, w)$ satisfying (91). This set is measurable with respect to $\mathcal{F} \times \mathcal{B}\left(\mathbb{N}^{\infty}\right) \times \mathcal{B}\left(\mathbb{R}^{n}\right)$, where $\mathbb{N}^{\infty}:=\mathbb{N} \times \mathbb{N} \times \ldots$ is the product of a countable number of copies of the discrete space $\mathbb{N}:=\{1,2, \ldots\}$ with the product topology. By virtue of Aumann's measurable selection theorem (see e.g. [10]), there exist measurable $v(\omega)=\left(N_{k}(\omega)\right)_{k=1}^{\infty}$ and $w(\omega)$ such that $(\omega, v(\omega), w(\omega)) \in A$ for almost all $\omega$. This yields (89) and (90) because $E|w(\omega)|=E \xi(\omega)=E \liminf \left|w_{N}(\omega)\right|$ by Fatou's lemma.

Let $C$ be a pointed closed cone in $\mathbb{R}^{n}$. We write $a \leq_{C} b$ if $b-a \in C$.

Proposition A.2 Let $p_{k}(\omega), k=1,2, \ldots$, be integrable random vectors with values in $C$ such that the sequence $\operatorname{Ep}_{k}(\omega)$ converges a.s. to a vector $q \in \mathbb{R}^{n}$. Then there exists a sequence 
of integer-valued random variables $1<k_{1}(\omega)<k_{2}(\omega)<\ldots$ and an integrable random vector $p(\omega)$ such that

$$
\lim _{l \rightarrow \infty} p_{k_{l}(\omega)}(\omega)=p(\omega)(\text { a.s. })
$$

and

$$
E p(\omega) \leq_{C} q
$$

Proposition A. 2 is a version of the multidimensional Fatou's lemma-an important result in measure theory first obtained in connection with applications in Mathematical Economics by Schmeidler [55] and then developed in various directions by Hildenbrand [33], Artstein [3], Balder and Hess [5], Cornet et al. [12], and others. Proposition A.2 extends Schmeidler's result from the case $C=\mathbb{R}_{+}^{n}$ to the case of a general cone $C$, and Proposition A.3 establishes an analogous fact for conditional expectations. For proofs of these propositions (under somewhat more general assumptions) see [6].

Let $\mathcal{G}$ be a sub- $\sigma$-algebra of $\mathcal{F}$ and let $C(\omega)$ be a pointed closed convex cone in $\mathbb{R}^{n}$ depending $\mathcal{G}$-measurably on $\omega$. A random vector $p(\omega)$ is said to be conditionally integrable (with respect to the $\sigma$-algebra $\mathcal{G}$ ) if $E[|p(\omega)| \mid \mathcal{G}]<\infty$ (a.s.), where $|\cdot|$ stands for a norm in $\mathbb{R}^{n}$.

Proposition A.3 Let $p_{k}(\omega), \omega \in \Omega, k=1,2, \ldots$, be conditionally integrable random vectors such that $p_{k}(\omega) \in C(\omega)$ (a.s.) and the conditional expectations $E\left[p_{k}(\omega) \mid \mathcal{G}\right]$ converge a.s. to a random vector $q(\omega)$. Then there exists a sequence of integer-valued random variables $1<k_{1}(\omega)<k_{2}(\omega)<\ldots$ and a conditionally integrable random vector $p(\omega)$ such that

$$
\lim _{l \rightarrow \infty} p_{k_{l}(\omega)}(\omega)=p(\omega)(\text { a.s. })
$$

and

$$
E[p(\omega) \mid \mathcal{G}] \leq C(\omega) q(\omega)(\text { a.s. })
$$

\section{References}

1. Algoet, P.H., Cover, T.M.: Asymptotic optimality and asymptotic equipartition properties of log-optimum investment. Ann. Prob. 16, 876-898 (1988)

2. Amir, R., Evstigneev, I.V., Schenk-Hoppé, K.R.: Asset market games of survival: a synthesis of evolutionary and dynamic games. Ann. Finance 9, 121-144 (2013)

3. Artstein, Z.: A note on Fatou's lemma in several dimensions. J. Math. Econ. 6, 277-282 (1979)

4. Aumann, R.J.: Existence of competitive equilibria in markets with a continuum of traders. Econometrica 34, 1-17 (1966)

5. Balder, E.J., Hess, C.: Fatou's lemma for multifunctions with unbounded values. Math. Oper. Res. 20, 175-188 (1995)

6. Babaei, E., Evstigneev, I.V., Schenk-Hoppé, K.R.: A multidimensional Fatou lemma for conditional expectations, preprint arXiv:1811.00640 [math.DS] (2018)

7. Babaei, E., Evstigneev, I.V., Schenk-Hoppé, K.R.: Log-optimal and rapid paths in von Neumann-Gale dynamical systems. J. Math. Anal. Appl. 481 (2020) (forthcoming)

8. Bahsoun, W., Evstigneev, I.V., Taksar, M.I.: Rapid paths in von Neumann-Gale dynamical systems. Stochastics 80, 129-142 (2008)

9. Breiman, L.: Optimal gambling systems for favorable games. Proceedings of the 4th Berkeley Symposium on Mathematical Statistics and Probability, vol. I, pp. 65-78. University of California Press, Berkeley (1961)

10. Castaing, C., Valadier, M.: Convex Analysis and Measurable Multifunctions, Lecture Notes in Math., vol 580, Springer, Berlin (1977) 
11. Clark, S.A.: Competitive prices for a stochastic input-output model with infinite time horizon. Econ. Theory 35, 1-17 (2008)

12. Cornet, B., Topuzu, M., Yildiz, A.: Equilibrium theory with a measure space of possibly satiated consumers. J. Math. Econ. 39, 175-196 (2003)

13. Cover, T.M.: Shannon and investment, IEEE information theory society newsletter, Special Golden Jubilee Issue, Summer, pp. 10-11 (1998)

14. Cover, T., Thomas, J.: Elements of Information Theory, 2nd edn. Wiley, New York (2006)

15. Cvitanić, J., Karatzas, I.: Hedging and portfolio optimization under transaction costs: a martingale approach. Math. Finance 6, 133-165 (1996)

16. Dasgupta, S., Mitra, T.: Infinite-horizon competitive programs are optimal. J. Econ. 69, 217-223 (1999)

17. Dempster, M.A.H., Evstigneev, I.V., Schenk-Hoppé, K.R.: Volatility-induced financial growth. Quant. Finance 7, 151-160 (2007)

18. Dempster, M.A.H., Evstigneev, I.V., Taksar, M.I.: Asset pricing and hedging in financial markets with transaction costs: an approach based on the von Neumann-Gale model. Ann. Finance 2, 327-355 (2006)

19. Dynkin, E.B.: Some probability models for a developing economy. Sov. Math. Dokl. 12, 1422-1425 (1971)

20. Dynkin, E.B.: Stochastic concave dynamic programming. USSR Math. Sb. 16, 501-515 (1972)

21. Dynkin, E.B., Yushkevich, A.A.: Controlled Markov processes and their applications. Springer, New York (1979). Chapter 9 of this monograph presents the main results of the papers [19] and [20]

22. Evstigneev, I.V., Flåm, S.D.: Rapid growth paths in multivalued dynamical systems generated by homogeneous convex stochastic operators. Set-Valued Anal. 6, 61-82 (1998)

23. Evstigneev, I.V., Schenk-Hoppé, K.R.: Stochastic equilibria in von Neumann-Gale dynamical systems. Trans. Am. Math. Soc. 360, 3345-3364 (2008)

24. Evstigneev, I.V., Hens, T., Schenk-Hoppé, K.R.: Evolutionary finance. In: Hens, T., Schenk-Hoppé, K.R. (eds.) Handbook of Financial Markets: Dynamics and Evolution, pp. 507-566. Elsevier, Amsterdam (2009). Chapter 9

25. Evstigneev, I.V., Hens, T., Schenk-Hoppé, K.R.: Mathematical Financial Economics: A Basic Introduction. Springer, Switzerland (2015)

26. Evstigneev, I.V., Hens, T., Schenk-Hoppé, K.R.: Evolutionary behavioural finance. In: Haven, E. (ed.) Handbook of Post Crisis Financial Modelling, pp. 214-234. Palgrave MacMillan, London (2016)

27. Evstigneev, I.V., Zhitlukhin, M.V.: Controlled random fields, von Neumann-Gale dynamics and multimarket hedging with risk. Stochastics 85, 652-666 (2013)

28. Gale, D.: A closed linear model of production. In: Kuhn, H.W., Tucker, A.W., et al. (eds.) Linear Inequalities and Related Systems, pp. 285-303. Princeton University Press, Princeton (1956)

29. Gale, D.: On optimal development in a multi-sector economy. Rev. Econ. Stud. 34, 1-18 (1967)

30. Grimmett, G.R., Stirzaker, D.R.: Probability and Random Processes, 3rd edn. Oxford University Press, Oxford (2001)

31. Guasoni, P., Rásonyi, M., Schachermayer, W.: Consistent price systems and face-lifting pricing under transaction costs. Ann. Appl. Prob. 18, 491-520 (2008)

32. Hakansson, N.H.: Capital growth theory. In: Jarrow, R., Maksimovic, A.V., Ziemba, W.T. (eds.) Handbooks in Operations Research and Management Science, vol. 9, pp. 65-86. Elsevier, Amsterdam (1995). Finance, Chapter 3

33. Hildenbrand, W.: Core and Equilibria of a Large Economy. Princeton University Press, New Jersey (1974)

34. Kabanov, YuM, Safarian, M.: Markets with Transaction Costs. Mathematical Theory. Springer, Berlin (2009)

35. Kabanov, YuM: Hedging and liquidation under transaction costs in currency markets. Finance Stoch. 3, 237-248 (1999)

36. Kelly, J.L.: A new interpretation of information rate. Bell Syst. Tech. J. 35, 917-926 (1956)

37. Long, J.B.: The numeraire portfolio. J. Financ. Econ. 26, 29-69 (1990)

38. Jouini, E., Kallal, H.: Martingales and arbitrage in securities markets with transaction costs. J. Econ. Theory 66, 178-197 (1995)

39. MacLean, L.C., Thorp, E.O., Ziemba, W.T. (eds.): The Kelly Capital Growth Investment Criterion: Theory and Practice. World Scientific, Singapore (2010)

40. Makarov, V.L., Rubinov, A.M.: Mathematical Theory of Economic Dynamics and Equilibria. Springer, Berlin (1977)

41. Malinvaud, E.: Capital accumulation and efficient allocation of resources. Econometrica 21, 233-268 (1953)

42. Morishima, M.: Equilibrium, Stability and Growth: A Multisectorial Analysis. Oxford University Press, Oxford (1964)

43. Natanson, I.P.: Theory of Functions of a Real Variable. Ungar, New York (1961) 
44. Nikaido, H.: Convex Structures and Economic Theory. Academic Press, London (1968)

45. Peleg, B.: On competitive prices for optimal consumption plans. SIAM J. Appl. Math. 26, 239-253 (1974)

46. Platen, E.: A benchmark approach to finance. Math. Finance 16, 131-151 (2006)

47. Platen, E., Heath, D.: A Benchmark Approach to Quantitative Finance. Springer, New York (2006)

48. Poundstone, W.: Fortune's Formula: The Untold Story of the Scientific Betting System That Beat the Casinos and Wall Street. Hill and Wang Publishing, New York (2006)

49. Radner, R.: Paths of economics growth that are optimal with regard only to final states: a Turnpike theorem. Rev. Econ. Stud. 28, 98-104 (1961)

50. Radner, R.: Efficiency prices for infinite horizon production programmes. Rev. Econ. Stud. 34, 51-66 (1967)

51. Radner, R.: Balanced stochastic growth at the maximum rate. In: Contributions to the von Neumann Growth Model, Conference Proceedings, Institute for Advanced Studies, Vienna, 1970), Zeitschrift für Nationalökonomie vol. 1, pp. 39-53 (1971)

52. Rockafellar, R. T.: Monotone Processes of Convex and Concave type. In: Memoirs of Amer. Math. Soc. vol 77, American Mathematical Society, Providence (1967)

53. Saadoune, M., Valadier, M.: Extraction of a good subsequence from a bounded sequence of integrable functions. J. Convex Anal. 2, 345-357 (1995)

54. Schachermayer, W.: The fundamental theorem of asset pricing under proportional transaction costs in finite discrete time. Math. Finance 14, 19-48 (2004)

55. Schmeidler, D.: Fatou's lemma in several dimensions. Proc. Am. Math. Soc. 24, 300-306 (1970)

56. Von Neumann., J.: Über ein ökonomisches Gleichungssystem und eine Verallgemeinerung des Brouwerschen Fixpunktsatzes, In: Ergebnisse eines Mathematischen Kolloquiums, vol. 8, 1935-1936 (1937). (Franz-Deuticke, Leipzig and Wien), pp. 73-83. [Translated: A model of general economic equilibrium, Review of Economic Studies vol. 13, pp. 1-9 (1945-1946).]

Publisher's Note Springer Nature remains neutral with regard to jurisdictional claims in published maps and institutional affiliations. 